\title{
Human bone marrow- and adipose- mesenchymal stem cells secrete exosomes enriched in distinctive miRNA and tRNA species
}

Serena Rubina Baglio ${ }^{1,2^{*}}$, Koos Rooijers ${ }^{3}$, Danijela Koppers-Lalic ${ }^{2}$, Frederik J. Verweij ${ }^{2}$, M Pérez Lanzón², Nicoletta Zini ${ }^{4,5}$, Benno Naaijkens², Francesca Perut ${ }^{1}$, Hans W. M. Niessen² ${ }^{2}$ Nicola Baldini ${ }^{1}$ and D. Michiel Pegtel ${ }^{2^{*}}$

\begin{abstract}
Introduction: Administration of mesenchymal stem cells (MSCs) represents a promising treatment option for patients suffering from immunological and degenerative disorders. Accumulating evidence indicates that the healing effects of MSCs are mainly related to unique paracrine properties, opening opportunities for secretome-based therapies. Apart from soluble factors, MSCs release functional small RNAs via extracellular vesicles (EVs) that seem to convey essential features of MSCs. Here we set out to characterize the full small RNAome of MSC-produced exosomes.

Methods: We set up a protocol for isolating exosomes released by early passage adipose- (ASC) and bone marrow-MSCs (BMSC) and characterized them via electron microscopy, protein analysis and small RNA-sequencing. We developed a bioinformatics pipeline to define the exosome-enclosed RNA species and performed the first complete small RNA characterization of BMSCs and ASCs and their corresponding exosomes in biological replicates.

Results: Our analysis revealed that primary ASCs and BMSCs have highly similar small RNA expression profiles dominated by miRNAs and snoRNAs (together 64-71\%), of which 150-200 miRNAs are present at physiological levels. In contrast, the miRNA pool in MSC exosomes is only 2-5\% of the total small RNAome and is dominated by a minor subset of miRNAs. Nevertheless, the miRNAs in exosomes do not merely reflect the cellular content and a defined set of miRNAs are overrepresented in exosomes compared to the cell of origin. Moreover, multiple highly expressed miRNAs are precluded from exosomal sorting, consistent with the notion that these miRNAs are involved in functional repression of RNA targets. While ASC and BMSC exosomes are similar in RNA class distribution and composition, we observed striking differences in the sorting of evolutionary conserved tRNA species that seems associated with the differentiation status of MSCs, as defined by Sox2, POU5F1A/B and Nanog expression.
\end{abstract}

Conclusions: We demonstrate that primary MSCs release small RNAs via exosomes, which are increasingly implicated in intercellular communications. tRNAs species, and in particular tRNA halves, are preferentially released and their specific sorting into exosomes is related to MSC tissue origin and stemness. These findings may help to understand how MSCs impact neighboring or distant cells with possible consequences for their therapeutic usage.

\footnotetext{
*Correspondence: s.baglio@vumc.nl; d.pegtel@vumc.nl

'Laboratory for Orthopedic Pathophysiology and Regenerative Medicine,

Istituto Ortopedico Rizzoli, Bologna 40136, Italy

${ }^{2}$ Department of Pathology, Cancer Center Amsterdam, VU University Medical

Center, De Boelelaan 1117, 1081 HV Amsterdam, The Netherlands

Full list of author information is available at the end of the article
} 


\section{Introduction}

Mesenchymal stem cells (MSCs) are intensively studied because they exhibit unique biological properties in vivo that are exploited for the treatment of many pathological conditions, most notably bone defects, degenerative illnesses, and autoimmunity [1].

MSCs are adult multipotent stem cells with selfrenewal potential [2] that can differentiate into alternate phenotypes of the mesenchymal germ layer, namely osteoblasts, chondrocytes, and adipocytes [3]. The most common source of MSCs is the bone marrow [4, 5]; however, MSCs reside in many other tissues, notably adipose tissue, which is highly relevant because it is an easy accessible abundant source of stem cells [6]. Whether MSCs from different sources can be considered as the same cell type and whether distinct environments may influence their phenotype and function are still under debate $[7,8]$.

Recent advances suggest that the beneficial effects of MSCs derive from secreted factors rather than from their tissue intercalation and differentiation. The MSC secretome drives organ healing by inducing a shift from proinflammatory to anti-inflammatory cytokine production at the site of injury [9-11]. These observations support the development of cell-free, secretome-based therapies that circumvent the risks associated with stem cell-based therapies such as immune-mediated rejection, accumulation of genomic alterations, and senescenceinduced genetic instability [12-14], and might require simpler safety regulations compared with their cell counterparts for clinical use [11].

The interest behind the MSC secretome goes beyond its application in tissue repair. Indeed, MSCs are strong contributors to tumor growth and progression in different cancer types [15-17], although anti-tumor activities have also been reported underscoring their pleiotropic properties $[18,19]$. A unique aspect of MSCs is that they strongly respond to inflammatory signals causing homing to active tumor sites, where they provide paracrine survival, proangiogenic and immune-modulatory signals, similar to those that promote wound healing. Previous studies have focused on characterizing MSC-produced soluble factors (i.e. cytokines, chemokines, and growth factors). However, it is now clear that, in addition to soluble factors, extracellular vesicles (EVs) are a key instrument in cell-cell communication [20]. Among the many subtypes of EVs, endosome-derived exosomes have emerged as physiologically relevant and powerful components of the MSC secretome [11, 12, 21].

Exosomes are nano-sized EVs with remarkable physiological properties, originating through inward budding of the limiting membrane of late endosomes called multivesicular bodies (MVBs). Upon fusion of MVBs with the plasma membrane, exosomes are released into the extracellular milieu and can be either taken up by target cells residing in the microenvironment or carried to distant sites via biological fluids. Besides transporting characteristic protein and lipid signatures, exosomes package nucleic acids, most notably various RNA species with regulatory functions [22]. Arguably the most studied class of exosome-enclosed RNAs is the class of microRNAs (miRNAs), which function in repressing their target mRNAs in recipient cells in vitro [23-26] and in vivo [27]. However, we recently showed in B cells that miRNAs only account for a fraction of the exosomal RNA. Indeed, other noncoding transcripts, including repeats and structural RNAs, complete the exosomal RNA repertoire produced in B cells but also in other cell types [28,29].

MSC-derived vesicles own remarkable properties typical of functional MSCs. Kordelas et al. [11] demonstrated the clinical efficacy of MSC exosomes to treat therapy-refractory graft-versus-host disease. Moreover, MSC-EVs regulate neurite outgrowth [30], promote angiogenesis both in vitro and in vivo [31], reduce myocardial ischemia/reperfusion injury [21], and repair acute kidney injury $[32,33]$. Thus, it is reasonable to postulate that MSC-EVs transport key MSC-associated molecules which change the physiology of target cells in a specific manner. Proteomic analysis suggests that MSC-EVs or subclasses thereof contain critical surface markers and signaling molecules characteristic of the MSCs [34]. Moreover, prior quantitative PCR profiling analysis [35] showed that while some miRNAs are present both in MSCs and in their corresponding microvesicles, others are selectively represented.

To optimally understand and exploit the clinical potential of adult MSC-derived exosomes, it is important to define the relevant functional molecules they enclose. Comprehensive information on the complete RNA content of MSC exosomes is currently not available, and whether adult MSCs from different sources share similar small RNA repertoires or whether their content is different remains unknown.

Here we describe the first comprehensive deepsequencing analysis of the small RNA profile of exosomes released by adult MSCs from two different sources: adipose-derived MSCs (ASCs) and bone marrow-derived MSCs (BMSCs). Our analysis of the exosomal content is useful for understanding how MSCs impact their microenvironment in resident niches and upon homing to damaged and inflamed tissues, which may have consequences for their therapeutic usage.

\section{Materials and methods Cell culture}

Human adipose tissue samples from elective plastic surgery were obtained from the Department of Plastic Surgery of Tergooi Hospital after the approval of the Medical 
Ethical Committee of the VUmc (METC, Amsterdam, the Netherlands) and written informed consent. Adipose tissue was processed within 24 hours as described previously [36]. Briefly, adipose tissue was minced using a surgical scalpel and digested with $0.1 \%$ collagenase A (Roche Mannheim, Germany) in phosphate-buffered saline (PBS) $1 \%$ bovine serum albumin (BSA; Roche Diagnostics) under continuous shaking for 45 minutes at $37^{\circ} \mathrm{C}$. After Ficoll density separation (Lymphoprep; Axis-Shield, Oslo, Norway) cells were seeded at a density of 100,000 cells $/ \mathrm{cm}^{2}$. The bone marrow of patients undergoing hip replacement was obtained from the Rizzoli Orthopedic Institute after the approval of the Comitato Etico dell'Istituto Ortopedico Rizzoli (Bologna, Italy) and written informed consent. Mononuclear cells were isolated by Ficoll Hystopaque gradient (SigmaAldrich, Milan, Italy) and seeded at a density of 250,000 cells $/ \mathrm{cm}^{2}$. After 4 days, nonadherent cells were removed and fresh medium was added to the cultures. ASCs and BMSCs were expanded in alpha-minimum essential medium ( $\alpha$-MEM; Lonza, Breda, The Netherlands) containing $100 \mathrm{U} / \mathrm{ml}$ penicillin, $100 \mu \mathrm{g} / \mathrm{ml}$ streptomycin (Gibco, Bleiswijk, the Netherlands), and $10 \%$ fetal bovine serum (FBS) or $5 \%$ platelet lysate (PL) [37] and $10 \mathrm{U} / \mathrm{ml}$ heparin (Leo Pharma, Amsterdam, the Netherlands), in a humidified atmosphere of $5 \% \mathrm{CO}_{2}$ at $37{ }^{\circ} \mathrm{C}$. The expression of typical MSC surface markers was analyzed by fluorescence-activated cell sorting (FACS), and the ability of the MSCs to undergo osteogenic differentiation was assessed by Alizarin red staining upon induction with ascorbic acid, dexamethasone, and $\beta$-glycerophosphate [38].

\section{Exosome isolation}

MSC exosomes were collected from approximately $3.2 \times$ $10^{7}$ cells at early passages (passages $2-3$ ). Once MSC cultures reached $70 \%$ confluence, cells were cultured for $24-$ 48 hours in $\alpha$-MEM containing exosome-depleted FBS or PL. Exosome-depleted FBS and PL were obtained by overnight centrifugation at $70,000 \times g$ at $4{ }^{\circ} \mathrm{C}$. Exosomes were isolated as described previously [39]. Briefly, MSC conditioned medium was centrifuged twice at $500 \times g$ for 10 minutes, twice at $2000 \times g$ for 15 minutes and twice at $10,000 \times g$ for 30 minutes. The supernatant was then transferred to Ultra-Clear tubes and centrifuged at 70,000 $\times g$ for 1 hour at $4{ }^{\circ} \mathrm{C}$ in a SW32Ti rotor (Beckman Coulter Inc., Woerden, The Netherlands). The exosome-containing pellet was washed with PBS and centrifuged at 70,000 $\times g$ for 1 hour. The pellet was then carefully resuspended in $200 \mu \mathrm{l}$ PBS and used immediately or stored at $-80^{\circ} \mathrm{C}$.

\section{Confocal laser scanning microscopy}

For confocal laser scanning microscopy analysis, MSCs were seeded on poly-L-lysine-coated (Sigma-Aldrich) coverslips, fixed with $4 \%$ paraformaldehyde, permeabilized with $0.1 \%$ Triton-X 100 and blocked with PBS $10 \%$ FBS (30 minutes). Slides were incubated with the primary antibodies against CD63 (BD Biosciences, Breda, The Netherlands) or EEA1 (Cell Signaling, Leiden, The Netherlands) and then with rabbit antimouse fluorescein isothiocyanate (FITC) antibody (DAKO, Heverlee, Belgium) for 30 minutes at room temperature. LysoTracker red (Molecular Probes, Bleiswijk, The Netherlands) was incubated with living cells before fixation. All stainings were imaged with a Leica DMRB microscope (Leica, Son, The Netherlands). Images were obtained through sequential scanning with the pinhole set at $1 \mathrm{AE}$ (standard). Fluorophores were excited using $488 \mathrm{~nm}$ (FITC) and $561 \mathrm{~nm}$ (Alexa594) laser lines.

\section{Western blotting}

For western blot analysis, cells were lysed with RIPA buffer containing protease inhibitor cocktail (Roche), and the protein concentration was determined by BCA assay (Pierce, Etten-Leur, The Netherlands). Cell lysates and exosome preparations diluted in sample buffer were run on a $10 \%$ SDS gel and blotted on a nitrocellulose membrane (GE Healthcare, Eindhoven, The Netherlands). Membranes were incubated with monoclonal antibodies against CD63, CD81, or cytochrome C (BD Biosciences) and horseradish peroxidase-conjugated rabbit anti-mouse secondary antibody (DAKO). Gels for CD63 and CD81 detection were run under nonreducing conditions.

\section{Transmission electron microscopy}

Cell pellets were fixed with $2.5 \%$ glutaraldehyde (Merck KGaA, Darmstadt, Germany) in phosphate buffer for 2 hours, post fixed with $1 \%$ osmium tetroxide, dehydrated in a graded series of ethanol, and embedded in Epon (Electron Microscopy Sciences, Hatfield, PA,USA). Ultrathin sections were stained with uranyl acetate and lead citrate. Exosome preparations were mixed with an equal volume of $4 \%$ paraformaldehyde (Sigma) in phosphate buffer. Then $5 \mu \mathrm{l}$ solution were deposited on 200 mesh Formvar-carbon-coated electron microscopy (EM) nickel grids and left to adsorb for 20 minutes at room temperature. Samples were fixed with $1 \%$ glutaraldehyde (Merck) in phosphate buffer, contrasted with uranyl oxalate (pH 7.0), and embedded in a mixture of $4 \%$ uranyl acetate and $2 \%$ methyl cellulose ( $25 \mathrm{cps}$; Sigma) in a 1:9 ratio on ice. Grids were then removed with stainless steel loops and the excess fluid was blotted with filter paper to ensure an appropriate thickness of the methyl cellulose film. After drying, grids were examined with a Zeiss EM109 transmission electron microscope (Zeiss, Oberkochen, Germany). Images were captured using a Nikon digital camera Dmx 1200F (Nikon 
Corporation, Tokyo, Japan), and ACT-1 software (Nikon Corporation).

\section{RNA isolation, RT-PCR, and generation of libraries for small RNA sequencing}

Total RNA was isolated using Trizol Reagent (Invitrogen, Breda, The Netherlands) as described previously [39]. Exosome preparations were pretreated with RNase A (SigmaAldrich) at a final concentration of $400 \mathrm{ng} / \mu \mathrm{l}$ at $37^{\circ} \mathrm{C}$ for 1 hour to degrade unprotected RNAs. The RNA quantity and purity were assessed with the Agilent 2100 Bioanalyzer system (Agilent, Amstelveen, The Netherlands). The expression analyses of differentiation and stemness-related genes were carried out using SYBR Green PCR master mix (Roche) in a LightCycler 480 real-time PCR system (Roche). Results were normalized with respect to glyceraldehyde 3-phosphate dehydrogenase (GAPDH) according to the $\Delta \Delta \mathrm{Ct}$ method [40]. cDNA libraries for sequencing were prepared using the TruSeq Small RNA Sample Preparation Kit (Illumina, Eindhoven, The Netherlands) following the manufacturer's instructions. Amplified cDNA constructs were purified on $6 \%$ PAGE gel and DNA molecules corresponding to 15-90 nucleotide transcripts were excised, eluted from gel, and concentrated by ethanol precipitation. Libraries were validated on the Bioanalyzer using the High Sensitivity DNA Chip (Agilent) and equimolarly pooled for the sequencing run. Sequencing was performed on a HiSeq 2000 (Illumina) paired end 100 cycle (PE100) run.

\section{Assignment of features to reads}

Adapter sequences were trimmed from the 3 ' ends of raw data using cutadapt (v1.1) [41] and the parameters "-O 12 -e 0.25 ". Trimmed reads were aligned to the human genome (build hg19) using bowtie (v2.0.6) [42], and multiple valid alignments per read were reported (up to 50) using the parameters "-seed 42 -gbar 100 -D 10 -R 2 -L 20 -N 0 -i C,1 -k 50 -score-min L,0,-0.4". For each read, only the alignments with the best score were used in subsequent analyses.

Several sources of genome annotation were used. GENCODE v.15 [43] was used, but transcripts with a total length larger than 120 were excluded, as well as genes of type "sense_intronic", "sense_overlapping", and "miRNA". For miRNAs, annotations from miRBase (v.19) [44] were used, for both primary and mature transcript annotations. tRNA annotations were obtained from GENCODE/tRNA and were supplemented with metadata from GtRNAdb [45]. piRNA annotations from the piRNA database [46] were used while collapsing overlapping annotations into clusters. Repeat annotations and metadata from RepeatMasker were obtained from the UCSC (University of California Santa Cruz) genome browser (27 March 2013) [47].
Using the (possibly multiple) alignments per read, and the annotations described before, the set of (possibly partially) overlapping genes was determined. In the case that the set of genes all belonged to a certain "featuretype" (i.e. RNA type), the featuretype was assigned to that read. In the case that the featuretype was ambiguous, the following steps were taken. If the length of the read was larger than 25 nucleotides, the featuretype "miRNA, processed" was eliminated; otherwise, the featuretype "miRNA, premature" was eliminated. If the length of the read was larger than 32 nucleotides, the featuretype "piRNA" was eliminated. If at this point the featuretype could be determined unambiguously, this featuretype was assigned. Otherwise, if there were two possible featuretypes, of which one was a repeat, the nonrepeat featuretype was assigned to the read. If this was not the case, and the read had two or more possible alignments, all featuretypes which were indicated by only one alignment were eliminated. Again, if at this point the featuretype could be determined unambiguously, this featuretype was assigned. In other cases, where the featuretype was not determined unambiguously, the read was designated as "Ambiguous" (Figure S1 in Additional file 1).

For further analyses, the alignments were processed and tables enumerating the featuretype, unique sequence, and count were created. This process enabled the analyses of sequence lengths split out by featuretype, and the analyses of fractions of RNA types per sample. For the analysis of differential expression of specific miRNA, the featuretype of miRNAs was further refined to resolve the miRNA name. Analogously, for detailed analysis of tRNAs and repeats, the featuretypes of these two classes were further refined to include the tRNA anti-codon and repeat family, respectively.

\section{Correlations of samples}

Correlations were only performed on genes which had at least a total number of 15 reads over the cell samples, or five reads over the exosome samples. Counts were normalized using the trimmed-mean-of-M-values normalization. Log values were calculated, and log values over zero were imputed per sample, by taking the lowest nonzero normalized value, dividing by two, and taking the $\log$ of that value (i.e. zero counts were imputed by an estimation of the lower limit of detection). Pearson correlation coefficients between the samples were calculated. In heatmaps, genes were clustered by their standardized euclidean distance, and samples were clustered by their correlation coefficients.

\section{tRNA/mRNA 3' UTR complementarity analysis}

A nonredundant list of tRNA fragment sequences was made from the 20 most abundant tRNA fragments per 
sample. A BLAST database of mRNA 3' UTRs was prepared using GENCODE v15 transcript annotations (only for transcripts having a complete CDS annotation). tRNA fragment sequences were searched for complementarity to 3' UTRs using BLAST (blastn v.2.2.25), with parameters allowing only ungapped, complementary hits ("-u T -S 2"), seed length 6 , and an adjusted effective search space size which was set to the total genomic length spanned by the 3' UTRs used in the database (to adjust for overlapping 3' UTRs originating from the same genomic sequence). The maximum allowed $e$ value was 1.0. For each hit, the conservation of the genomic region that matched to the tRNA was inspected using phyloP 100-way data (obtained from UCSC tracks, dd. 20MAR2015). Additionally, the conservation of the matching region relative to the shortest overlapping 3' UTR was calculated.

\section{Statistical analyses}

All statistical analyses were carried out in $\mathrm{R}$ software (http://www.r-project.org/). Differential expression was determined using the exactTest routine from the edge $R$ package [48] and common library dispersions. Counts were normalized using the supplied trimmed-mean-of$\mathrm{M}$-values algorithm, except in the analysis of high-level feature types (e.g. "miRNA", "piRNA", "repeat"), in which case total library sizes were used to normalize.

Research was carried out in compliance with the Helsinki Declaration and all experimental protocols were approved by the Ethical Committee of the VU University Medical Center and of the Rizzoli Orthopaedic Institute.

\section{Results}

Mesenchymal stem cells from adipose tissue and bone marrow release exosome-like EVs enriched in small RNAs To study the mesenchymal stem cell-released EVs we isolated primary adult MSCs from human bone marrow (BMSCs, $n=4$ ) and adipose tissue (ASCs, $n=3$ ) (Figure S2 in Additional file 1). Early passage MSCs (passages $0-2$ ) were expanded in exosome-depleted FBS or PL to support in vitro expansion [36].

The MSC endosomal compartment was analyzed by immunofluorescence and by EM. The immunofluorescent staining showed high punctate expression of CD63, which was mainly localized in nonacidic vesicles in the perinuclear region of the cells, as determined by lysotracker (Fig. 1a, top left). The early endosome antigen A1 (EEA1) staining highlighted the presence of numerous early endosomes distributed throughout the cell body (Fig. 1a, bottom left). However, despite the expression of endosomal markers, the ultrastructure of the stem cells revealed relatively low numbers of late endosomes with internal vesicular structures, suggestive of MVB-like compartments and/or secretory lysosomes. These compartments had a diameter of about
$500 \mathrm{~nm}$ and enclosed 40-100 $\mathrm{nm}$ intraluminal vesicles (Fig. 1a, right). The low abundance of MVB-like organelles detectable by EM suggests that the high amount of CD63 observed in the perinuclear space of MSCs is associated with intracellular membranes not related to MVBs.

EVs were isolated by differential centrifugation as described previously [39] and their purity was confirmed by EM and western blotting for CD63 and CD81. Cytochrome $\mathrm{C}$ was assessed to exclude contamination by apoptotic bodies (Fig. 1b). Although differential centrifugation is the most commonly used method to isolate exosomes from culture supernatant [49], it is not possible to rule out the presence of other types of EVs in the exosome preparations when using this procedure. However, because our preparations obtained by ultracentrifugation seem to be enriched for exosomes in terms of size, shape, and tetraspanin content, we consider these MSC-EVs as "exosomes". To exclude potential contamination of MSC-exosome preparations by PL or FBS-derived EVs, we analyzed CD63 and CD81 in exosome-depleted culture media subjected to differential centrifugation. Since no CD63 was detectable, we concluded that our preparations contained mainly MSCderived exosomes (Figure S3A in Additional file 1).

To define the small RNA composition of the MSC exosomes we degraded any unprotected RNA in exosome preparations by adding exogenous RNase A. Subsequently, we isolated cellular and exosomal RNA, which was subjected to Bioanalyzer profiling. The small RNA profile of exosomes revealed characteristic peaks between 20 and 70 nucleotides, suggestive of the presence of miRNAs and tRNAs. The size distribution in cells was more heterogeneous and included longer transcripts (Fig. 1c). We constructed cDNA libraries of MSC cellular and exosomal RNA molecules with a length ranging between approximately 15 and 90 nucleotides (Figure S3B in Additional file 1).

We observed that early passage primary MSCs contain relatively few MVBs, which suggests that these cells secrete relatively few exosomes compared with other cell types [23, 50, 51]. However, MSC-released exosomes incorporate a small RNA population that is protected from exogenous RNases.

\section{Mesenchymal stem cells and their exosomes have a different RNA composition}

The sequencing of the libraries yielded a total of 25 million reads. The cell samples showed a wide distribution of read length, with a predominant peak around $22 \mathrm{nu}$ cleotides (Fig. 2a), regardless of the tissue origin and donor. Exosomes released by ASCs had a major peak between 31 and 36 nucleotides, while BMSC exosome samples showed two different profiles and were therefore classified into subtypes (BMSC I and BMSC II). 
A
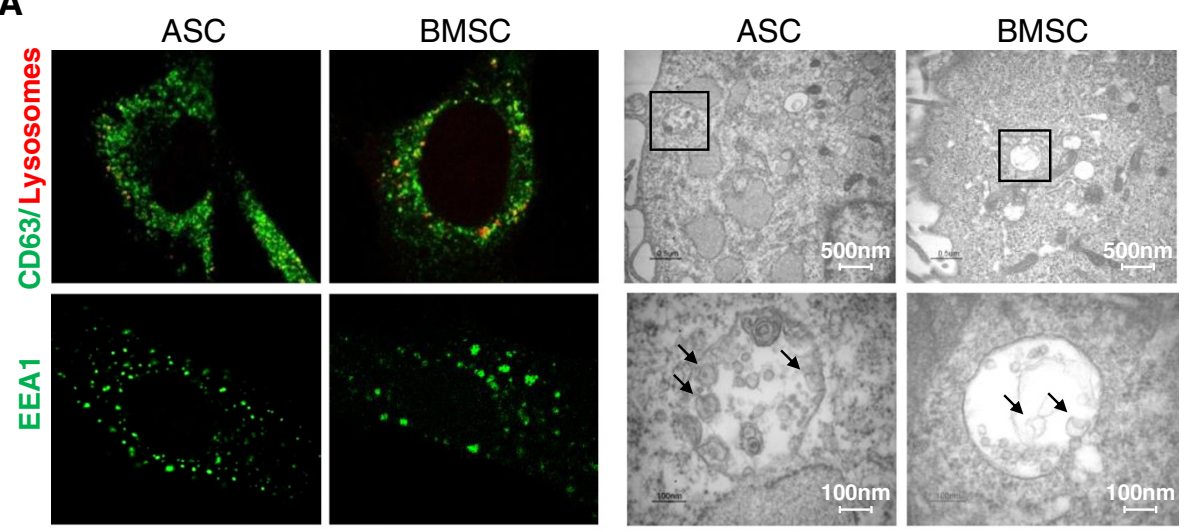

B
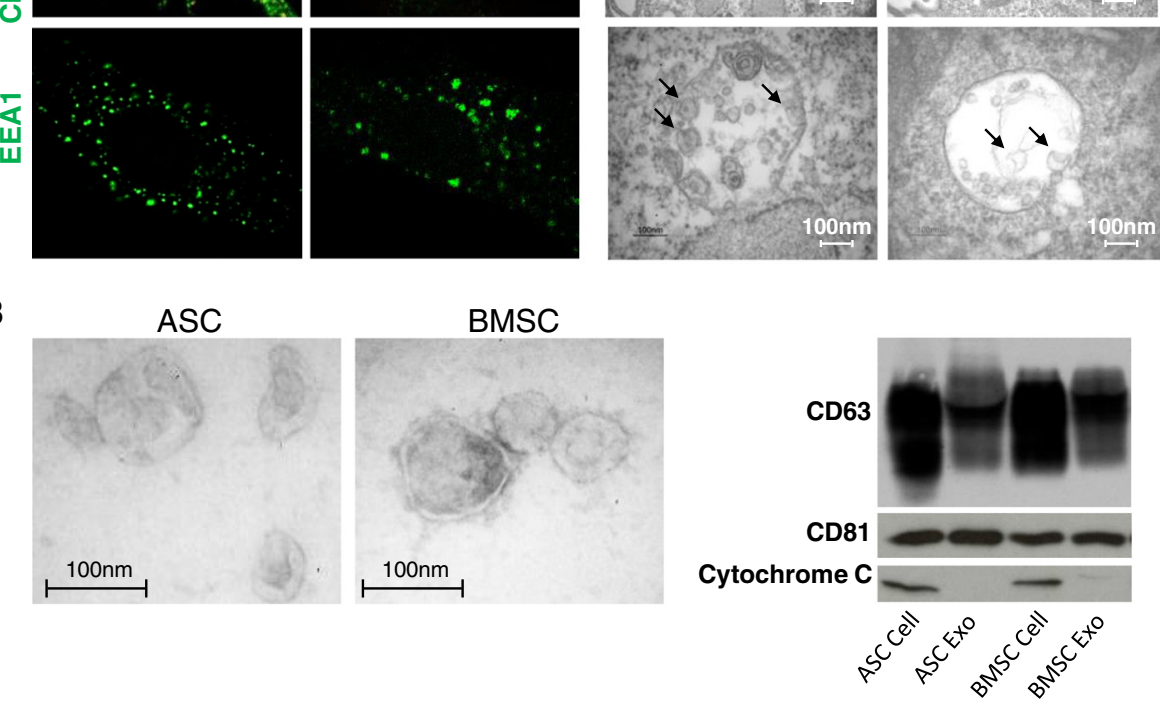

C

Cell

Exo
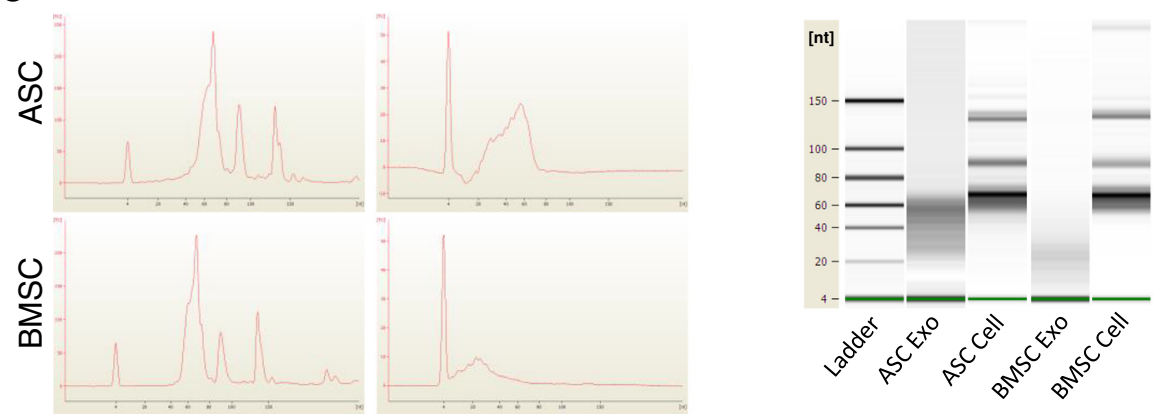

Fig. 1 ASCs and BMSCs contain relatively few MVBs and release exosome-like vesicles enriched in small RNAs. a Immunofluorescent staining of CD63 (top left) and EEA1 (bottom left), and ultrastructure of MVB-like endosomes (right) in ASCs and BMSCs. b Transmission electron microscopy micrographs of exosomes isolated from ASCs and BMSCs (left); and western blot for CD63, CD81, and cytochrome C in cells and corresponding exosomes (right). c Bioanalyzer small RNA profile of cells and exosomes showing enrichment of 20-70 nucleotide small RNAs in exosomes. ASC adipose-derived mesenchymal stem cell, BMSC bone marrow-derived mesenchymal stem cell, EEA1 early endosome antigen A1, exo exosome

BMSC I exosomes had a length distribution similar to that of ASC, while BMSC II exosomes displayed additional peaks at 15 and 70 nucleotides. In order to assess the variability among samples we performed an unsupervised hierarchical clustering analysis. Clustering analysis revealed a strong similarity among MSC samples irrespective of the stem cell source (Fig. 2b). Interestingly, however, when looking at the exosome preparations, ASC samples cluster tightly together, while BMSC I and BMSC II exosome samples appear dissimilar from each other. In accordance, the correlation analysis (Fig. 3a) indicated a strong correlation among cellular samples $(0.87<r<0.94)$. This correlation decreased when comparing exosome libraries: the Pearson coefficient within the BMSC I and BMSC II subtypes was 0.90 and 0.87 respectively, while it ranged from 0.66 and 0.73 between the two subgroups. Among ASC exosomes the coefficient ranged from 0.84 to 0.88 , while collectively was between 0.65 and 0.84 between ASC and BMSC exosomes.

The relatively low correlation between the two subtypes of BMSC exosomes prompted us to investigate whether the producing cells may represent different differentiation stages. Because MSCs can spontaneously undergo osteogenic differentiation during in-vitro expansion [52], we analyzed the expression of early osteogenic 


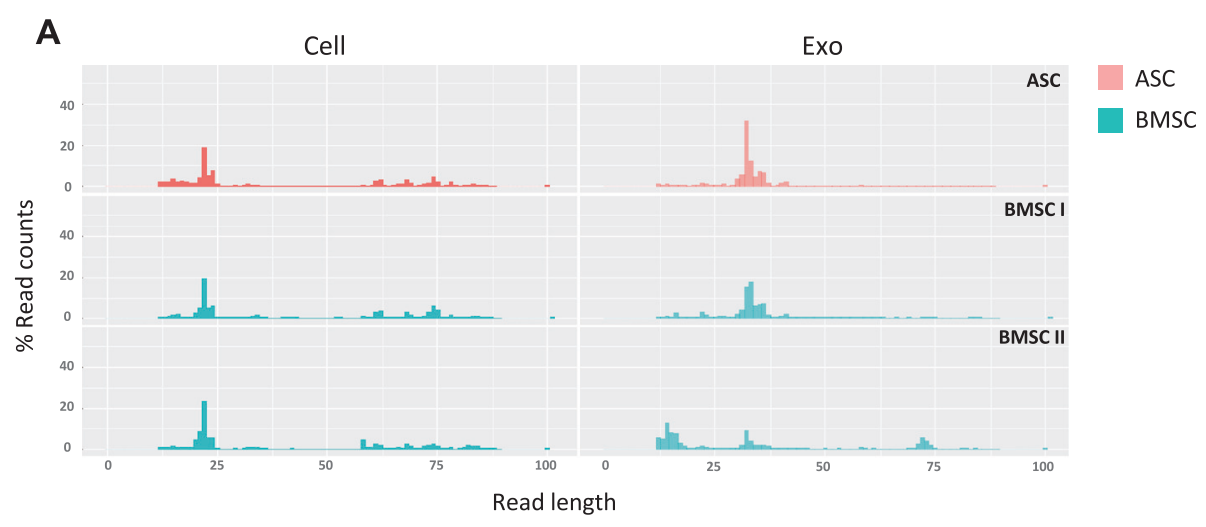

B

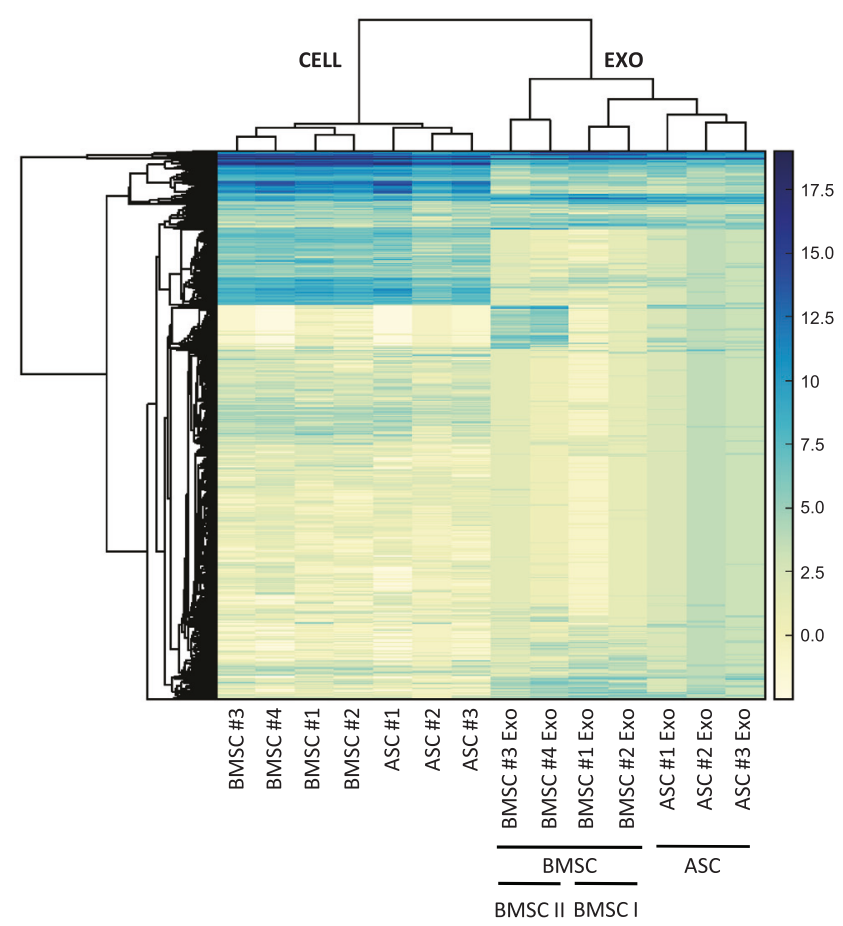

Fig. 2 MSCs and their exosomes display a different small RNA composition. a Length distribution of RNAseq aligned reads in ASCs and BMSCS and corresponding exosomes (one representative donor). b Unsupervised hierarchical clustering analysis of MSCs and exosomes based on the total small RNA content. ASC adipose-derived mesenchymal stem cell, BMSC bone marrow-derived mesenchymal stem cell, exo exosome

markers-i.e. Runx2, alkaline phosphatase (ALP), and collagen type 1 alpha 1 (COL1A1) -and of the stemnessrelated genes SOX2, POU5F1A/B, and Nanog by quantitative PCR (Fig. 3b). While we did not observe differences in the expression of bone-related genes, BMSC II subtype cells had elevated expression of Sox2, POU5F1A/B, and Nanog as compared with the other subtypes (ANOVA test, Fisher's PLSD correction: $p<0.05$ BMSC II vs. ASC and BMSC II vs. BMSC I for Sox2, and POU5F1A/B, and BMSC II vs. ASC for Nanog). The different expression levels of the pluripotency genes reflecting the stemness of the MSCs might explain the dissimilarity in exosome small RNA composition.
The correlation matrix analysis (Fig. 3a) highlighted a weak correlation between cells and corresponding exosomes $(r \leq 0.57)$. This implies that exosomes do not strictly reflect the RNA composition of the cells of origin, but selectively incorporate a variety of RNA species. Indeed, we observed enrichment of distinct RNA classes in cells while others were overrepresented in exosomes (Fig. 4a, b). In all MSC samples, miRNAs and small nucleolar RNAs (snoRNAs) were the most abundant classes of RNA in the cells, although their proportion was variable independently of the tissue source (19-49\% and $21-49 \%$, respectively) (Fig. 4a). These two classes together accounted for $64-71 \%$ of the entire cellular 
A

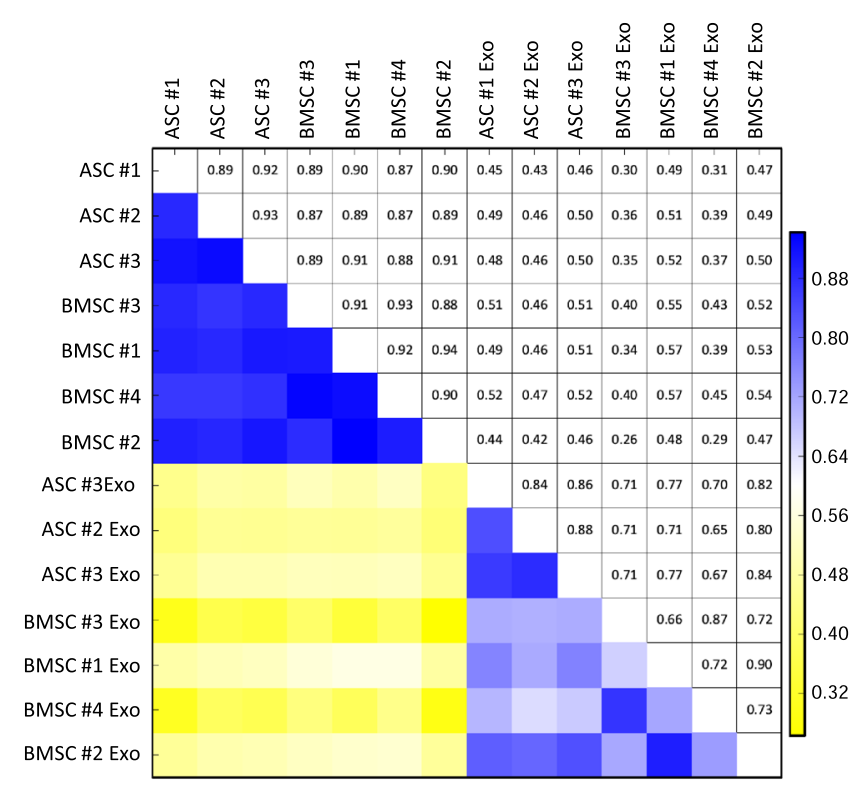

B

Runx2

ALP

COL1A1
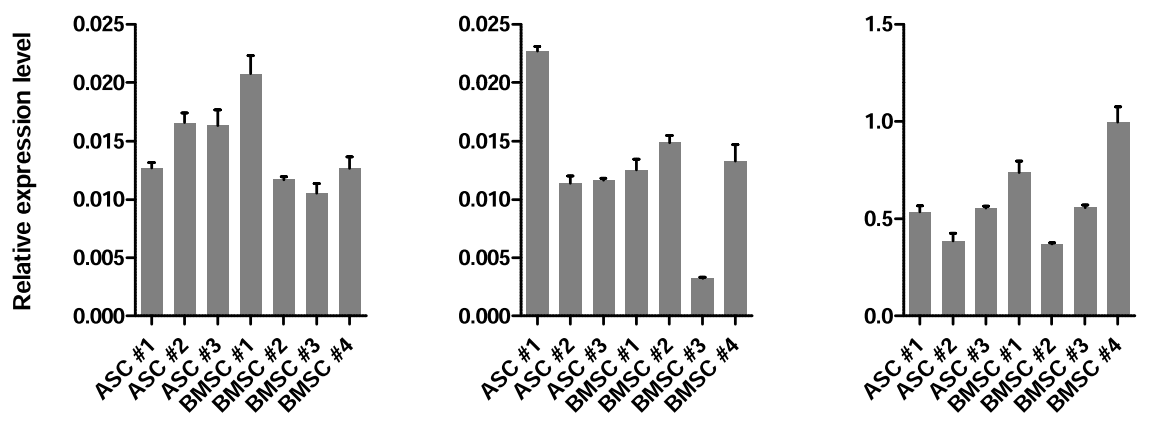

Sox2

PoU5F1A/B

Nanog
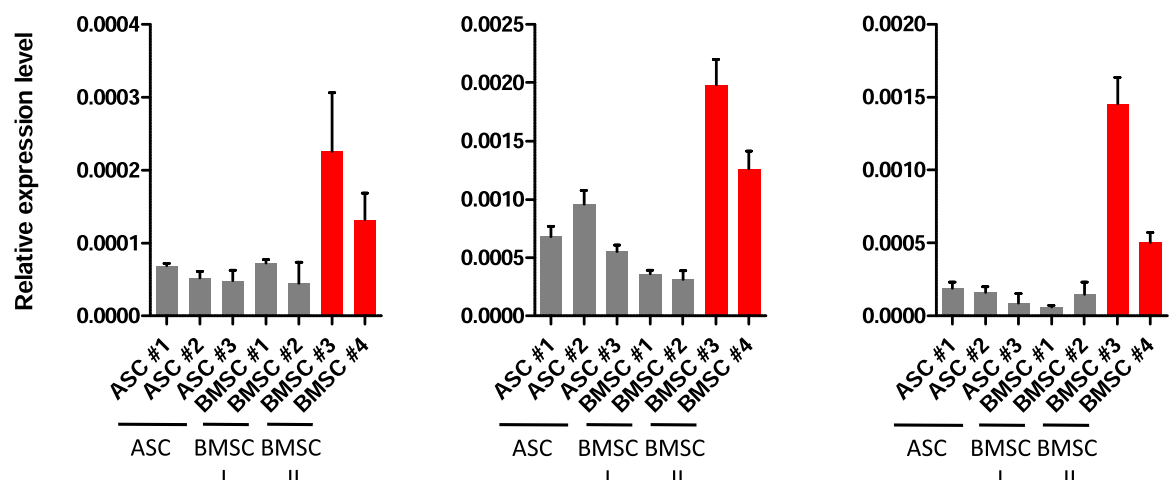

Fig. 3 Exosomes released by MSCs at different stages of differentiation correlate moderately with each other. a Correlation matrix of MSC and exosome samples. b Relative expression levels of early osteogenic differentiation (top) and multipotency (bottom) genes analyzed by quantitative PCR. Data were normalized to GAPDH. BMSC II express higher levels of Sox2 (ANOVA test: $p=0.006$; Fisher's PLSD test: $p=0.02$ BMSC II vs. ASC, $p=0.03$ BMSC II vs. BMSC I), POU5F1A/B (ANOVA test: $p=0.01$; Fisher's PLSD test: $p=0.03$ BMSC II vs. ASC, $p=0.01$ BMSC II vs. BMSC I) and Nanog (ANOVA test: $p=0.03$; Fisher's PLSD test: $p=0.056$ BMSC II vs. ASC) compared with the other subtypes. ALP alkaline phosphatase, ANOVA analysis of variance, ASC adipose-derived mesenchymal stem cell, BMSC bone marrow-derived mesenchymal stem cell, COLIA1 collagen type 1 alpha 1, exo exosome, GAPDH glyceraldehyde 3-phosphate dehydrogenase, Fisher's PLSD Fisher's Protected Least Significant Difference 


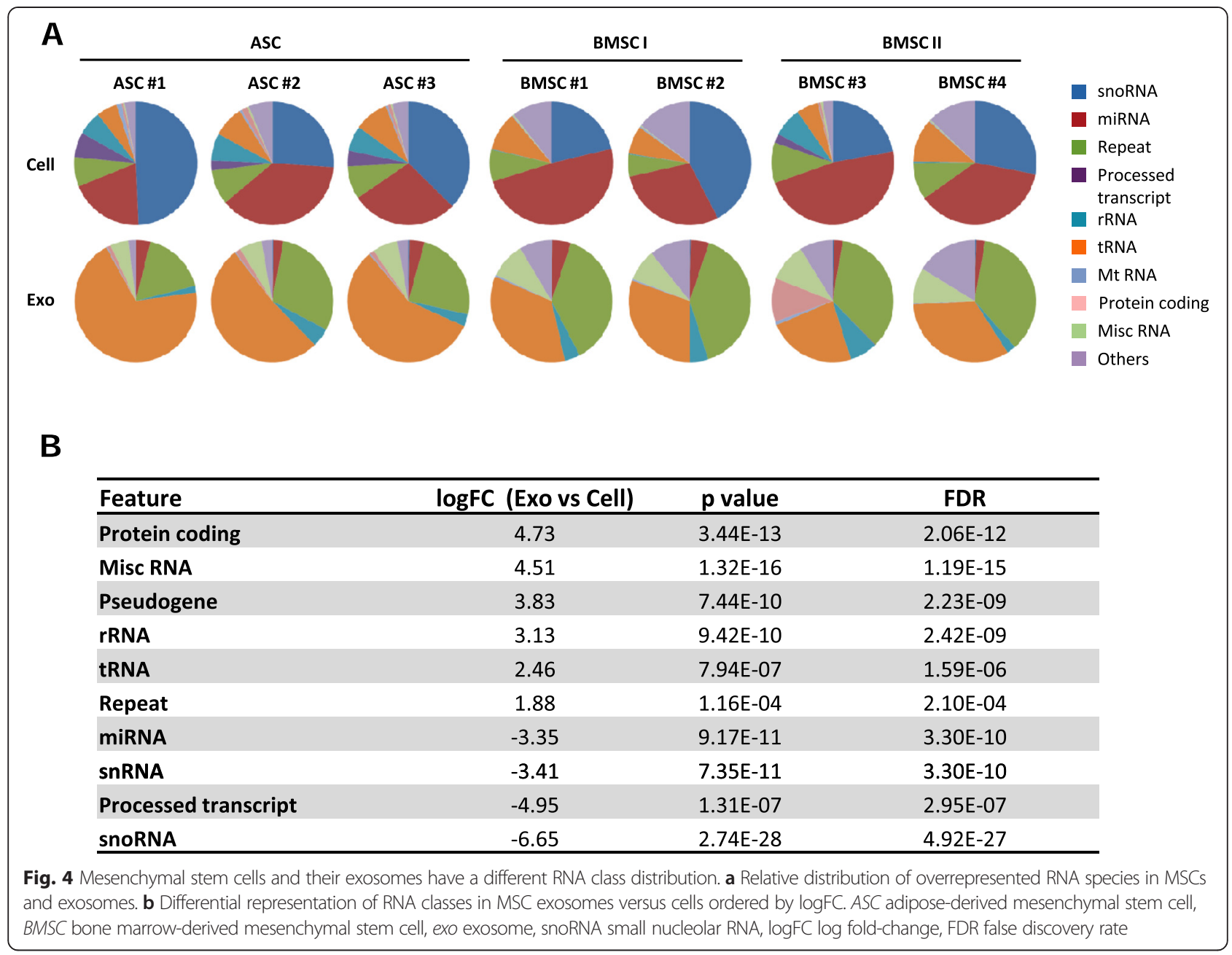

small RNA pool, followed by repeats (6-11\%), tRNA (5-11\%), and rRNAs (up to $8 \%$ ). In contrast, exosome libraries were highly enriched in the class of tRNAs, which represented $>50 \%$ of total small RNAs in adipose-derived exosomes and $23-35 \%$ in bone marrow exosomes, and repeats, ranging from $17-30 \%$ in adipose exosomes to $24-40 \%$ in bone marrow exosomes (Fig. 4a). The dominance of tRNAs in exosomes is consistent with their function and abundance in the cellular cytoplasm. Other represented classes in exosomes were miscellaneous RNAs, rRNAs, and miRNAs, the latter representing only $2-5 \%$ of the small RNA repertoire. In sharp contrast with that observed in MSC libraries, snoRNAs only represented a very small proportion of the exosomal RNA content $(<0.6 \%)$.

In summary, our data indicate that miRNAs and snoRNAs are significantly enriched in the cells (miRNA: $\operatorname{logFC} 3.35$, FDR $3.3 \times 10^{-10}$; snoRNA: $\operatorname{logFC} 6.65$, FDR $4.9 \times 10^{-27}$ ) while tRNAs and repeats form a defined pool of RNAs heavily enriched in exosomes (tRNA: $\operatorname{logFC} 2.46$, FDR $1.59 \times 10^{-6}$; repeats: $\operatorname{logFC} 1.88$, FDR $\left.2.1 \times 10^{-4}\right)$, suggestive of preferential sorting and release. The length distribution (Fig. 2a) showed predominant peaks between 31 and 36 nucleotides in ASC and BMSC I exosomes and additional peaks at around 15 and 70 nucleotides in BMSC II exosomes, suggestive of the presence of full-length tRNA and tRNA fragments in exosomes. Since tRNAs and tRNA fragments are involved in translation regulation and RNA silencing, these observations may point to a physiological link between post-transcriptional regulation and exosome biogenesis in MSCs [53, 54].

\section{Mesenchymal stem cell exosomes selectively incorporate specific miRNAs}

A substantial proportion of the cellular small RNA content in MSCs (19-49\%) is miRNA. However, this class is underrepresented in exosomes $(2-5 \%$ of the total small RNA) (Fig. 4a). Because of this discrepancy we investigated whether cells and exosomes share similar miRNA content. 
In line with our previous findings on the total small RNA profile, unsupervised clustering analysis and correlation analysis based on the miRNA content only revealed high similarity within cellular samples $(0.89<r$ $<0.96)$, while exosome samples displayed greater variability $(0.75<r<0.90)$. Overall, the correlation between cells and exosomes appears weak (Fig. 5a; and Figure S4A in Additional file 1).

To assess whether miRNAs were present as precursors or fully processed transcripts we looked at the length distribution of the miRNA reads (Fig. 5b), and found that in all MSC subtypes, both in exosomes and corresponding cells, the vast majority of miRNA sequences ranged between 20 and 25 nucleotides. Accordingly, the sequencing coverage of the most represented miRNA genes (UCSC genome browser) (Fig. 5c) predominantly shows the presence of mature miRNAs mapping to the $5 p$ and/or 3p arms of the precursor.

We then examined the relative proportion of individual miRNAs in the repertoire of total miRNA reads (Fig. 6a; and Figure S4B in Additional file 1). Surprisingly, the five most abundant miRNAs (miR-486-5p, miR-10a-5p, miR-10b-5p, miR-191-5p, and miR-222-3p in ASC exosomes; and miR-143-3p, miR-10b-5p, miR486-5p, miR-22-3p, and miR-21-5p in BMSC exosomes) accounted for $43-59 \%$ of the total miRNA reads. To evaluate the relative distribution of miRNAs in cells and exosomes, we ranked cellular and exosomal miRNA based on the reads per million (rpm) values and compared the 20 most represented miRNAs in cells and exosomes (Table 1). miR-21-5p, miR-22-3p, miR-10b-5p, and miR-222-3p were among the most represented in both cells and exosomes; however, various miRNAs (shown in bold in Table 1) were only present either in the list of cellular or in the list of exosomal highly represented miRNAs. We next asked whether specific miRNAs may be preferentially excreted or retained in the cells. Figure $6 \mathrm{~b}$ shows the top four miRNAs overrepresented in exosomes compared with MSCs $(\operatorname{logFC}>7$; FDR $\left.<5 \times 10^{-15}\right)$. On the other hand, miR-34a-5p, miR$34 c-5 p$, miR-15a-5p, and miR-136-3p were significantly overrepresented in cells compared with exosomes $(\log \mathrm{FC}$ $>3$; FDR $<3 \times 10^{-6}$ ) (Fig. 6c). The relative abundance of these miRNAs is shown in Figure S4C in Additional file 1. Altogether, the nonrandom distribution of miRNAs is consistent with a sorting mechanism for disposal of "unused" small RNAs or for communication with the surrounding environment as shown in other cell types [55].

\section{tRNA-derived RNA fragments are highly represented in MSC exosomes}

The dramatic overrepresentation of tRNA sequences in MSC exosomes prompted us to investigate which tRNAs were the most represented and whether these could be functional processed transcripts or degradation products.

Our analysis revealed that generally the adult MSC subtypes have similar tRNA profiles irrespective of the tissue source (Fig. 7a, top). tRNA CTC (Glu) was highly represented both in ASCs and in BMSCs, accounting for $43-72 \%$ of the total tRNA reads. In order to exclude potential experimental biases leading to high representation of one specific tRNA sequence, we also analyzed the tRNA profile obtained by small RNAseq in an unrelated cell type-i.e. lymphoblastoid cells (LCLs) (Figure S5A in Additional file 1). Interestingly, we found a very distinct tRNA distribution in these cells suggesting that, similar to miRNAs, tRNA profiles may be useful as indicators of tissue origin. Overall, exosomal tRNA profiles appeared distinct from the cellular profiles (Figure S6 in Additional file 1) and displayed more intergroup variability (Fig. 7a, bottom). The five most prevalent tRNA sequences in MSC exosomes accounted for $87-97 \%$ of the total exosomal tRNA pool (consisting of 40-54 different acceptors). Interestingly, while in BMSCs and LCLs the most abundant tRNAs in cells and exosomes clearly correspond, the most abundant tRNA in ASC exosomes, tRNA GCC (Gly), only represented a small fraction (5\%) of the total cellular tRNA (Fig. 7a). Moreover, this tRNA was overrepresented in ASC exosomes compared with BMSC exosomes (logFC 3.8; FDR $1.1 \times 10^{-7}$ ) (Figure S5B in Additional file 1).

To investigate whether MSC libraries contain full-length tRNAs or processed transcripts we analyzed the length distribution of the tRNA reads (Fig. 7b). Surprisingly, both in cells and exosomes, tRNA fragments constitute a considerable fraction of the total tRNA. Although MSCs from both tissue origins display a broad range of tRNA lengths, exosomal tRNA sequences have specific fragment sizes. ASC exosomes and BMSC I exosomes mainly contained fragments of 30-35 nucleotides, whereas BMSC II exosomes also show a dominant peak at 70-75 nucleotides. Intrigued by these findings we zoomed into the genomic coverage and length distribution of the most represented tRNA genes (Fig. 7c; and Figure S5C in Additional file 1). Strikingly, while at the cellular level adipose and bone marrow MSCs contain the full-length form of the most abundant tRNA, tRNA CTC (Glu), exosomes released by ASC and BMSC I exosomes consistently display the 33nucleotide $5^{\prime}$ halves of the most abundant sequences (Fig. 7c; and Figure S5C in Additional file 1). BMSC II exosomes that are produced by cells with high expression of pluripotency factors seem to preferentially enclose the full-length form of tRNA CTC (Glu), and the 33nucleotide fragments of other abundant tRNA species (Figure S5C in Additional file 1). The remarkable differences in tRNA composition observed between adipose and bone marrow exosomes warrant future investigations 

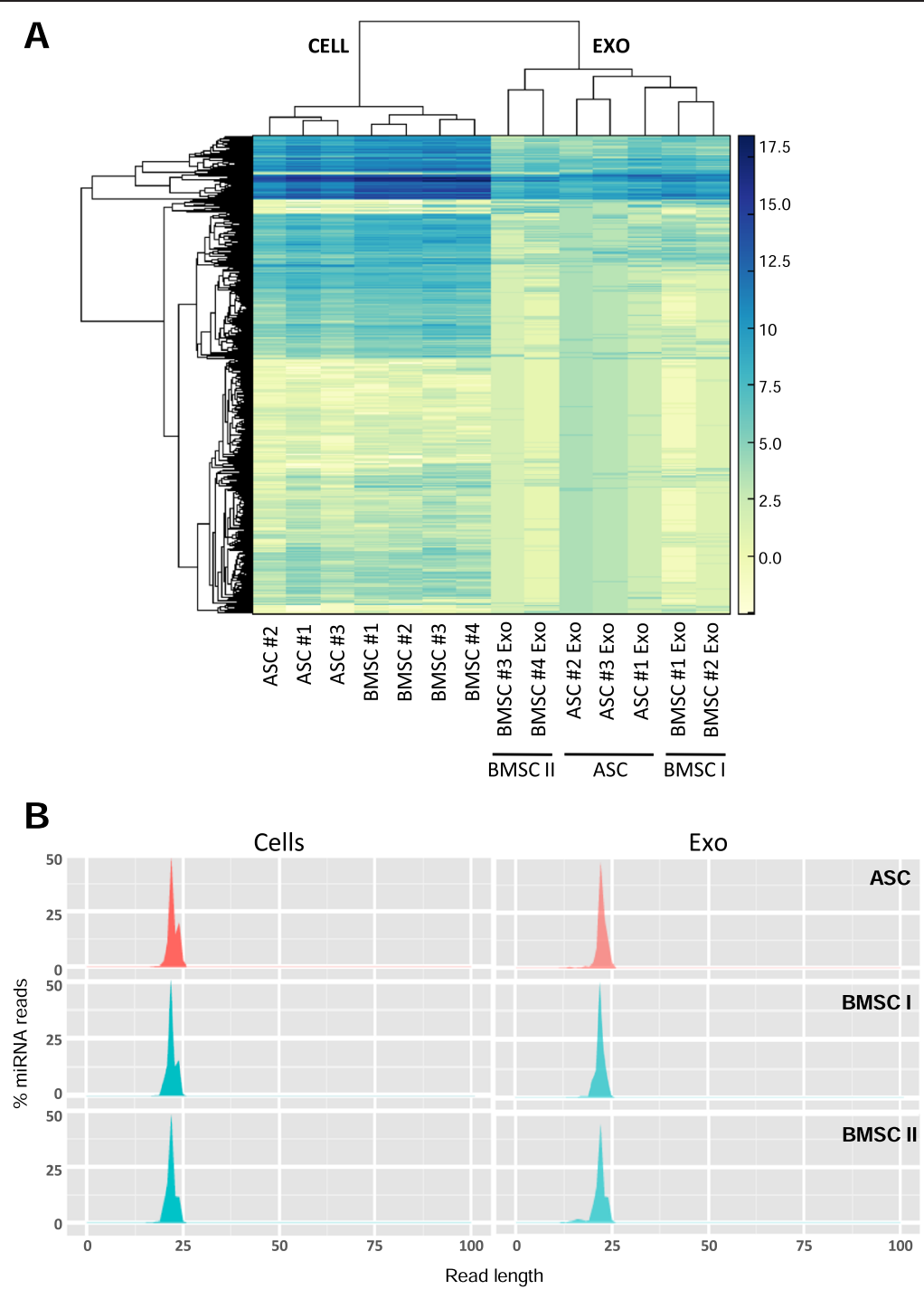

C

miR-486

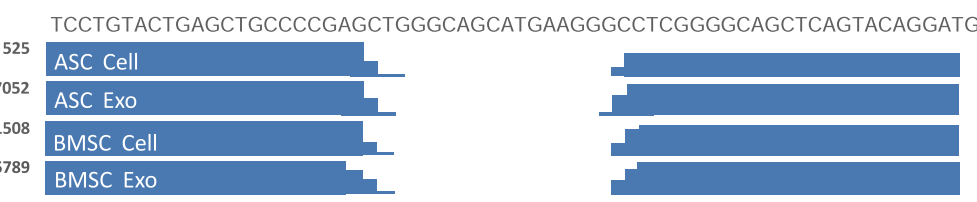

miR-222

CAGAGACCCAGTAGCCAGATGTAGCTGCTGATTACGAAAGACAGGATCTACACTGGCTACTGAGCCA

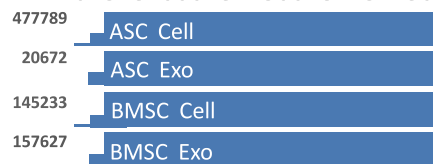

Fig. 5 MSCs and their exosomes display a different miRNA repertoire. a Unsupervised hierarchical clustering analysis of MSCs and their exosomes based on the miRNA content. $\mathbf{b}$ Length distribution of miRNA reads in ASC and BMSC I and BMSC II cells and exosomes (one representative donor). c Sequence coverage of highly represented miRNA genes (based on UCSC genome browser custom tracks) showing a predominant presence of mature forms (one or both miRNA arms) in MSCs and exosomes. Y axis indicates the normalized counts (rpm). ASC adipose-derived mesenchymal stem cell, BMSC bone marrow-derived mesenchymal stem cell, exo exosome 


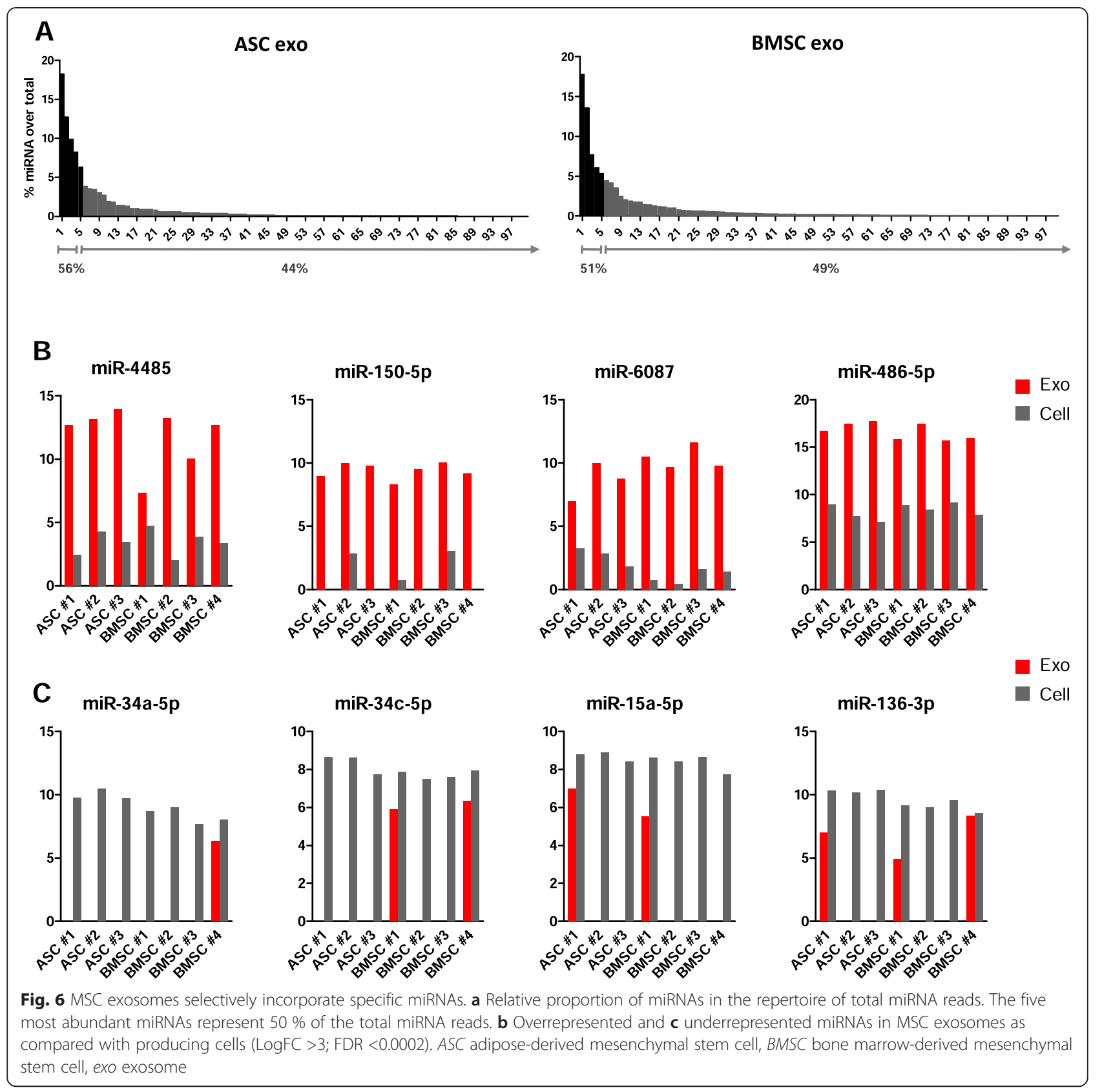

into the paracrine effects of adult stem cells from different sources and at different stages of differentiation.

Although the function of tRNA fragments and tRNA halves in mammalian cells is still largely unknown, a miRNA/siRNA (small interfering RNA)-like function for these tRNA species has been recently suggested [56]. Here we performed a bioinformatic analysis to evaluate the antisense complementarity of the most abundant tRNA species in cells and exosomes to the 3' UTRs of annotated protein-coding transcripts. We found a group of putative candidate targets (Table 2; and Additional file 2 ), many of which have undefined biological function.
Interestingly, among the potential targets we found factors involved in stem cell self-renewal and MSC differentiation, such as TFCP2L1, RUNX2, and SOX11, the transforming growth factor-beta (TGF $\beta$ ) signaling mediator SMAD3, and immune-related factors such as HHLA2, EMR2, and TRIM62.

\section{Discussion}

The administration of MSC-EVs is advantageous over cell-based therapy because it eliminates the safety concerns associated with the injection of multipotent cells into patients [12]. MSC-secreted exosomes have recently 
Table 1 Most abundant miRNAs in MSC exosomes and cells

\begin{tabular}{|c|c|c|c|c|}
\hline \multirow[t]{2}{*}{ Rank } & \multicolumn{2}{|c|}{ Most represented miRNA in exosomes } & \multicolumn{2}{|c|}{ Most represented miRNA in cells } \\
\hline & miRNA & rpm ASC exosomes & miRNA & rpm ASC cells \\
\hline 1 & miR-486-5p & 172,837 & miR-21-5p & 145,332 \\
\hline 2 & $\operatorname{miR}-10 a-5 p$ & 117,453 & $\operatorname{miR}-22-3 p$ & 92,902 \\
\hline 3 & miR-10b-5p & 104,447 & miR-10b-5p & 82,089 \\
\hline 4 & miR-191-5p & 92,545 & miR-222-3p & 74,725 \\
\hline 5 & miR-222-3p & 49,405 & miR-143-3p & 73,794 \\
\hline 6 & miR-22-3p & 37,205 & let-7a-5p & 71,686 \\
\hline 7 & let-7a-5p & 28,814 & miR-10a-5p & 44,303 \\
\hline 8 & miR-21-5p & 27,233 & miR-92a-3p & 33,827 \\
\hline 9 & $\operatorname{miR}-127-3 p$ & 27,071 & let-7f-5p & 33,248 \\
\hline 10 & miR-143-3p & 22,579 & let-7i-5p & 22,624 \\
\hline 11 & miR-99b-5p & 21,471 & miR-127-3p & 17,303 \\
\hline 12 & miR-100-5p & 18,836 & miR-148a-3p & 17,109 \\
\hline 13 & miR-92a-3p & 18,217 & miR-26a-5p & 16,127 \\
\hline 14 & let-7f-5p & 17,589 & miR-92b-3p & 14,852 \\
\hline 15 & miR-92b-3p & 16,124 & $\operatorname{miR}-21-3 p$ & 14,548 \\
\hline 16 & miR-26a-5p & 13,207 & miR-221-3p & 12,481 \\
\hline 17 & miR-146a-5p & 12,212 & $\operatorname{miR}-16-5 p$ & 11,544 \\
\hline 18 & miR-4485 & 10,656 & miR-100-5p & 11,180 \\
\hline 19 & miR-146b-5p & $10,, 558$ & $\operatorname{miR}-31-5 p$ & 9521 \\
\hline \multirow[t]{2}{*}{20} & miR-151a-3p & 10,262 & $m i R-411-5 p$ & 8051 \\
\hline & miRNA & rpm BMSC exosomes & miRNA & rpm BMSC cells \\
\hline 1 & miR-143-3p & 124,950 & miR-143-3p & 185,884 \\
\hline 2 & miR-10b-5p & 103,485 & miR-21-5p & 150,993 \\
\hline 3 & miR-486-5p & 91,274 & miR-22-3p & 105,358 \\
\hline 4 & miR-22-3p & 74,730 & let-7a-5p & 91,387 \\
\hline 5 & miR-21-5p & 47,445 & miR-10b-5p & 42,729 \\
\hline 6 & miR-222-3p & 46,094 & miR-222-3p & 38,076 \\
\hline 7 & miR-191-5p & 45,054 & miR-27b-3p & 35,496 \\
\hline 8 & miR-100-5p & 41,668 & let-7f-5p & 29,054 \\
\hline 9 & let-7a-5p & 38,486 & let-7i-5p & 21,993 \\
\hline 10 & miR-99b-5p & 29,011 & miR-26a-5p & 21,934 \\
\hline 11 & miR-92a-3p & 24,941 & miR-100-5p & 21,157 \\
\hline 12 & miR-127-3p & 21,319 & miR-127-3p & 14,836 \\
\hline 13 & let-7f-5p & 21,203 & $\operatorname{miR}-148 a-3 p$ & 12,789 \\
\hline 14 & miR-92b-3p & 20,938 & miR-92b-3p & 12,306 \\
\hline 15 & $\operatorname{miR}-423-5 p$ & 19,807 & miR-92a-3p & 11,840 \\
\hline 16 & miR-10a-5p & 14,716 & miR-191-5p & 11,384 \\
\hline 17 & miR-27b-3p & 13,604 & $\operatorname{miR}-21-3 p$ & 11,372 \\
\hline 18 & let-7i-5p & 11,997 & miR-125b-5p & 10,699 \\
\hline 19 & miR-28-3p & 10,554 & let-7b-5p & 9603 \\
\hline 20 & miR-125b-5p & 10,378 & miR-16-5p & 9583 \\
\hline
\end{tabular}

miRNAs present only in the list of cellular or in the list of exosomal miRNAs are highlighted in bold

ASC adipose-derived mesenchymal stem cell, BMSC bone marrow-derived mesenchymal stem cell, miRNA microRNA, MSC mesenchymal stem cell, rpm reads per million 
A

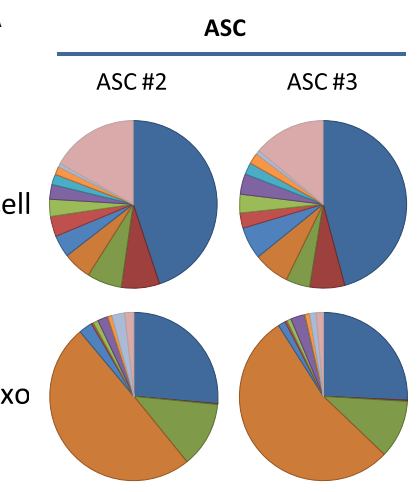

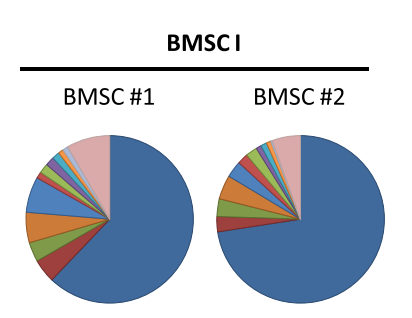
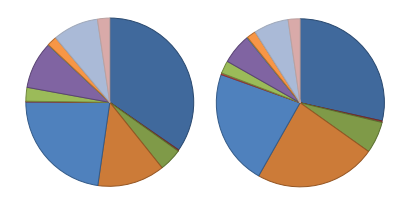
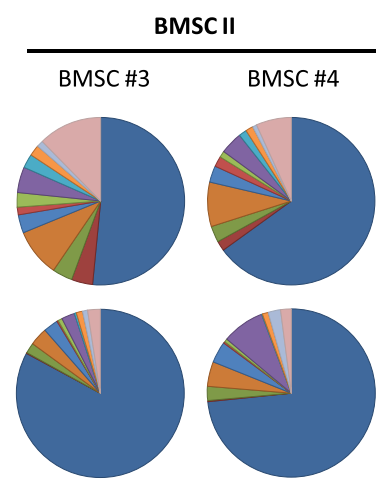

Exo

B

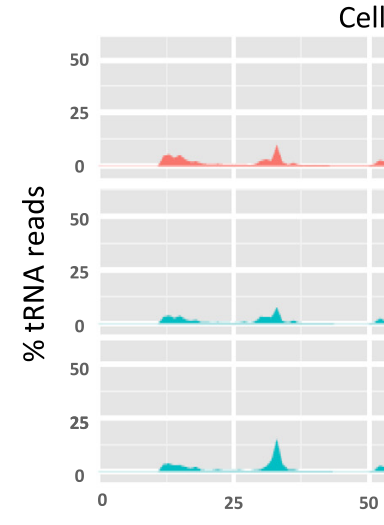

Cells
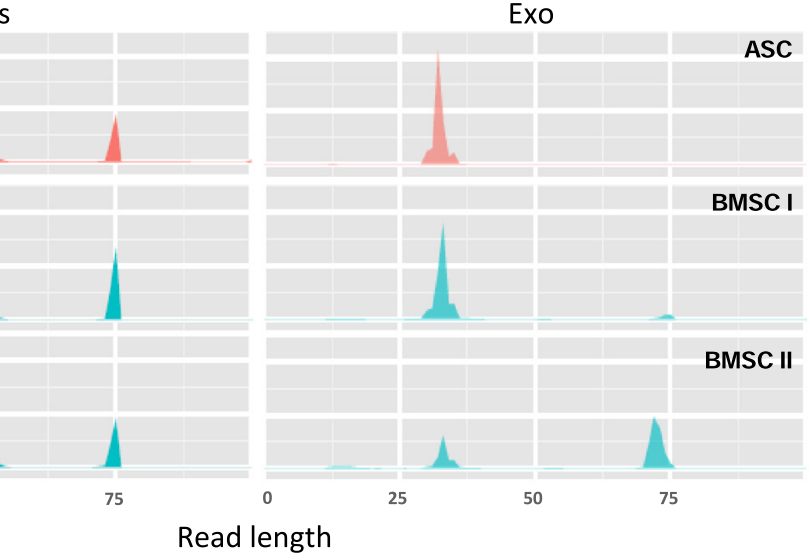

$\square$ ASC

BMSC

tRNA Lys; TTT

tRNA Glu; TTC

tRNA Gly; GCC

tRNA His; GTG

tRNA Gly; CCC

tRNA Asp; GTC

tRNA Val; CAC

tRNA Gln; CTG

tRNA Lys; CTT

tRNA Val; AAC

Others

Read length

\section{C}

tRNA CTC (Glu)
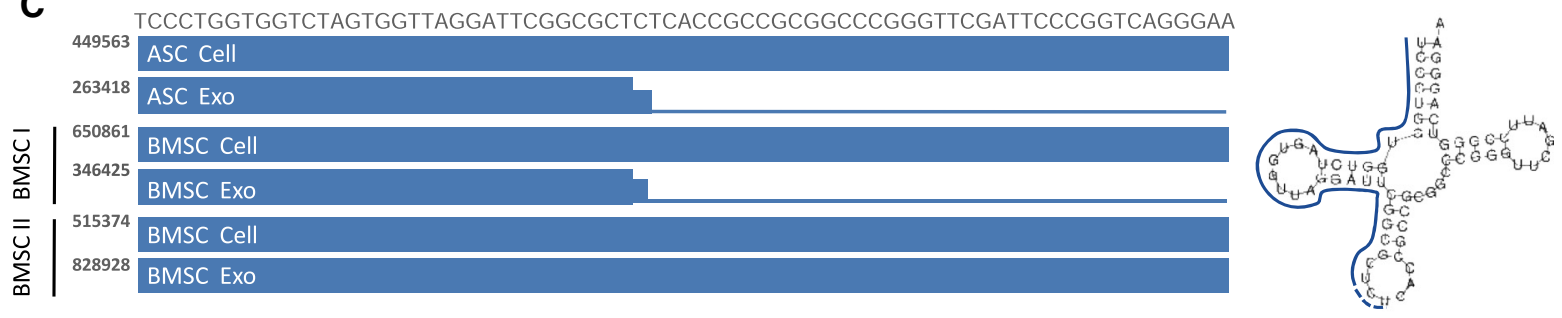

tRNA GCC (Gly)
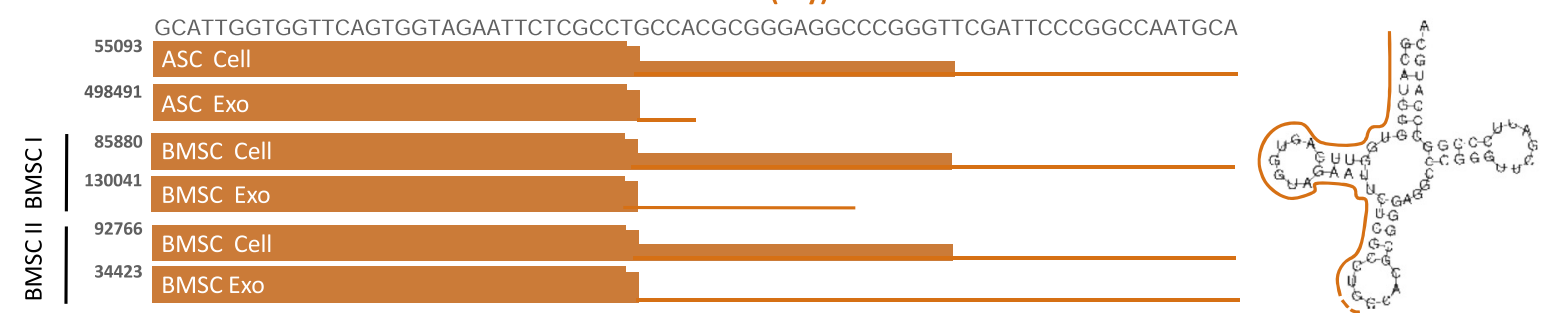

Fig. 7 tRNA-derived RNA fragments are highly represented in MSC exosomes. a Relative distribution of highly represented tRNAs in MSCs and respective exosomes. $\mathbf{b}$ Length distribution of tRNA sequencing reads in ASC and BMSC I and II cells and exosomes. c Sequence coverage of highly represented tRNA genes (based on UCSC genome browser custom tracks). Y axis indicates the normalized counts (rpm). ASC adipose-derived mesenchymal stem cell, BMSC bone marrow-derived mesenchymal stem cell, exo exosome 
Table 2 Complementarity analysis of tRNA species to 3' untranslated regions of protein-coding genes

\begin{tabular}{|c|c|c|c|c|c|c|c|}
\hline Query & Query sequence & Hit length & $e$ value & Bitscore & Gene name & Conservation value & $\begin{array}{l}\text { Normalized } \\
\text { conservation value }\end{array}$ \\
\hline tRNA; Glu; CTC & $\begin{array}{l}\text { AGTGGTTAGGATTCGGC } \\
\text { GCTCTCACCGCCGCGGCCC }\end{array}$ & 14 & 0.19 & 28.2 & GOLGA6A & 0.510 & 0.970 \\
\hline \multirow[t]{11}{*}{ tRNA; Glu; TTC } & $\begin{array}{l}\text { TCCCTGGTGGTCTAGTG } \\
\text { GCTAGGATTCGGCGCTIT }\end{array}$ & 24 & 0.012 & 32.2 & TFCP2L1 & 0.146 & 1.067 \\
\hline & & 14 & 0.19 & 28.2 & NELF & -0.303 & 0.962 \\
\hline & & 17 & 0.74 & 26.3 & KIAA0513 & -0.367 & 1.013 \\
\hline & & 13 & 0.74 & 26.3 & ZBTB45 & -0.447 & 0.950 \\
\hline & & 13 & 0.74 & 26.3 & HHIPL1 & -1.103 & 0.808 \\
\hline & & 13 & 0.74 & 26.3 & HSP90AA1 & -0.248 & 0.982 \\
\hline & & 13 & 0.74 & 26.3 & TULP4 & 1.937 & 1.345 \\
\hline & & 13 & 0.74 & 26.3 & SMAD3 & 0.259 & 1.001 \\
\hline & & 13 & 0.74 & 26.3 & RPS19 & -0.474 & 0.984 \\
\hline & & 13 & 0.74 & 26.3 & ZNF662 & 0.140 & 0.990 \\
\hline & & 13 & 0.74 & 26.3 & AC104841.2 & 2.810 & 1.542 \\
\hline \multirow[t]{28}{*}{ tRNA; Gly; GCC } & $\begin{array}{l}\text { GCATGGGTGGTTCAG } \\
\text { TGGGAGAATTCTCGCCT }\end{array}$ & 18 & 0.19 & 28.2 & PHF13 & 0.042 & 0.914 \\
\hline & & 14 & 0.19 & 28.2 & KDSR & -0.243 & 0.904 \\
\hline & & 14 & 0.19 & 28.2 & PLAGL2 & 0.634 & 0.881 \\
\hline & & 13 & 0.74 & 26.3 & NOX5 & -0.231 & 0.982 \\
\hline & & 13 & 0.74 & 26.3 & EPM2AIP1 & 0.373 & 0.996 \\
\hline & & 13 & 0.74 & 26.3 & GTF3C1 & -0.476 & 1.095 \\
\hline & & 13 & 0.74 & 26.3 & GPR110 & 0.293 & 1.053 \\
\hline & & 13 & 0.74 & 26.3 & TPI1 & 0.241 & 0.971 \\
\hline & & 13 & 0.74 & 26.3 & PPP2R1B & -0.302 & 0.875 \\
\hline & & 13 & 0.74 & 26.3 & LUZP2 & 0.125 & 0.998 \\
\hline & & 13 & 0.74 & 26.3 & VPS41 & -0.243 & 0.977 \\
\hline & & 13 & 0.74 & 26.3 & SSR1 & 0.187 & 1.025 \\
\hline & & 13 & 0.74 & 26.3 & RUNX2 & 1.358 & 0.956 \\
\hline & & 13 & 0.74 & 26.3 & MAP1LC3B & -0.254 & 0.890 \\
\hline & $\begin{array}{l}\text { GCATGGGTGGTTCA } \\
\text { GTGGTAGAATTCTCGCCG }\end{array}$ & 14 & 0.19 & 28.2 & GCM1 & 0.127 & 1.032 \\
\hline & & 17 & 0.74 & 26.3 & SLC2A13 & 0.507 & 0.983 \\
\hline & & 13 & 0.74 & 26.3 & GTF3C1 & -0.476 & 1.095 \\
\hline & & 13 & 0.74 & 26.3 & HHLA2 & -0.075 & 1.004 \\
\hline & & 13 & 0.74 & 26.3 & TPI1 & 0.241 & 0.971 \\
\hline & & 13 & 0.74 & 26.3 & PEG10 & -0.126 & 0.806 \\
\hline & & 13 & 0.74 & 26.3 & EMR2 & -0.166 & 0.954 \\
\hline & & 13 & 0.74 & 26.3 & SSR1 & 0.187 & 1.025 \\
\hline & & 11 & 0.7 & 22.3 & THAP5 & 0.155 & 0.979 \\
\hline & & 11 & 0.7 & 22.3 & THAP5 & 0.393 & 1.029 \\
\hline & $\begin{array}{l}\text { GCATGGGTGGTTCA } \\
\text { GTGGTAGAATTCTCGCCTG }\end{array}$ & 14 & 0.19 & 28.2 & GCM1 & 0.127 & 1.032 \\
\hline & & 17 & 0.74 & 26.3 & SLC2A13 & 0.507 & 0.983 \\
\hline & & 13 & 0.74 & 26.3 & GTF3C1 & -0.476 & 1.095 \\
\hline & & 13 & 0.74 & 26.3 & HHLA2 & -0.075 & 1.004 \\
\hline
\end{tabular}


Table 2 Complementarity analysis of tRNA species to 3' untranslated regions of protein-coding genes (Continued)

\begin{tabular}{|c|c|c|c|c|c|c|}
\hline & 13 & 0.74 & 26.3 & TPI1 & 0.241 & 0.971 \\
\hline & 13 & 0.74 & 26.3 & PEG10 & -0.126 & 0.806 \\
\hline & 13 & 0.74 & 26.3 & EMR2 & -0.166 & 0.954 \\
\hline & 13 & 0.74 & 26.3 & SSR1 & 0.187 & 1.025 \\
\hline & 11 & 0.73 & 22.3 & THAP5 & 0.155 & 0.979 \\
\hline & 11 & 0.73 & 22.3 & THAP5 & 0.393 & 1.029 \\
\hline \multirow[t]{13}{*}{$\begin{array}{l}\text { GCATTGGTGGTTCAGTGGTAGAATTCT } \\
\text { CGCCTGCCACGCGGGAGGCCCGGGT }\end{array}$} & 20 & 0.012 & 32.2 & SOX11 & 0.180 & 0.752 \\
\hline & 14 & 0.19 & 28.2 & GCM1 & 0.127 & 1.032 \\
\hline & 17 & 0.74 & 26.3 & SLC2A13 & 0.507 & 0.983 \\
\hline & 17 & 0.74 & 26.3 & FAM57B & -0.158 & 0.849 \\
\hline & 13 & 0.74 & 26.3 & HHLA2 & -0.075 & 1.004 \\
\hline & 13 & 0.74 & 26.3 & C1orf159 & -0.663 & 0.984 \\
\hline & 13 & 0.74 & 26.3 & KLC2 & -0.224 & 0.835 \\
\hline & 13 & 0.74 & 26.3 & PEG10 & -0.126 & 0.806 \\
\hline & 13 & 0.74 & 26.3 & DRG2 & -0.468 & 0.817 \\
\hline & 13 & 0.74 & 26.3 & VPRBP & 1.514 & 0.913 \\
\hline & 13 & 0.74 & 26.3 & TRIM62 & -0.098 & 0.921 \\
\hline & 13 & 0.74 & 26.3 & EMR2 & -0.166 & 0.954 \\
\hline & 13 & 0.74 & 26.3 & ANXA8L2 & 2.926 & 1.223 \\
\hline
\end{tabular}

been shown to improve therapy-refractory graft-versushost disease [11] and to promote organ healing in various preclinical models [21, 32, 33]. Comprehensive characterization of these vesicles is therefore a critical step in understanding their biological activity to maximize clinical utility.

MSCs have been described before as prolific producers of exosomes when compared with some other cell types [57]. However, this conclusion was based on the use of myc-transformed (immortalized) human embryonic stem cell-derived MSCs [58], which was required to overcome the limited in-vitro expansion potential. Because immortalized cells may secrete pro-oncogenic material via EVs [59], the clinical usefulness of these vesicles is uncertain. In addition, immortalized MSCs may produce EVs with an altered content, casting additional doubt as to whether these EVs are representative of their natural counterparts [60]. We show here that unmanipulated MSCs in culture produce few EVs with exosome characteristics. Accordingly, MSCs in culture possess relatively few prototypical MVBs compared with most cell types analyzed thus far in our laboratory [23, 50]. Although MSC exosomes have the same morphology as exosomes from B-cell blasts and carry typical marker proteins, they may differ in compartmentalization, biogenesis, and therefore RNA composition. Moreover, we presume that factors such as tissue origin (adult or embryonic) and stemness could influence both exosome production and content.

The very low intragroup and intergroup variability among MSC samples indicates that donor-specific characteristics and the tissue-specific microenvironment do not significantly influence the small RNA expression profile of the cells. However, important differences emerged when comparing EV preparations. The variability between ASC and BMSC EVs suggests that the tissue-specific microenvironment might influence the exosomal sorting of the MSCs. The intragroup variability indicates that cell-intrinsic factors, such as the differentiation status of the cells, might dictate which signals are conferred by the cells, as previously reported for cytokines and growth factors [61, 62].

Although most studies show that miRNAs only represent a small fraction of exosomal RNA [28, 63, 64], miRNAs transferred via exosomes can be functional in repressing their target in vitro and in vivo [23-27]. In our analysis, we found that the five most abundant miRNAs in MSC exosomes accounted for $50 \%$ of the total miRNA reads. Thus, specific miRNAs present in high amounts might have physiological effects. In a previous study, Chen et al. [65] showed that particles secreted by human embryonic stem cell-derived MSCs are enriched in pre-miRNAs. In contrast, our study reveals that adult MSC exosomes mainly contain mature transcripts. 
While it is possible that the EVs released by embryonic and adult MSCs preferentially enclose different miRNA forms, conditioned medium as a whole presumably contains heterogeneous populations of RNA when compared with exosomes purified by differential ultracentrifugation, as analyzed in this study. Possibly, the pre-miRNAs are not released in association with exosomes but are incorporated into vesicles of different nature. Although there is a substantial similarity between the most represented miRNAs in ASC and BMSC exosomes, their relative proportions are different, raising the possibility that ASCs and BMSCs might deliver different information into their microenvironments. Some of these miRNAs have been implicated in MSC biology [66]. miR-486 is involved in ASC replicative senescence [67], miR-143 has been related to the immune modulatory function of MSCs [68], miR$10 \mathrm{a}$ and miR-22 are important regulators of MSC differentiation $[69,70]$, and miR-10b promotes the migration of mouse BMSCs [71]. The release of these miRNAs by ASCs and BMSCs could play a role in stem cell niche maintenance by controlling and fine-tuning proliferation, differentiation, and homing. In addition, multiple miRNAs highly represented in adult MSC exosomes regulate cell cycle progression and proliferation (miR-191, miR-222, miR-21, let-7a), and modulate angiogenesis (miR-222, miR-21, let-7f) and endothelial cell differentiation (miR6087) [72-74]. The uptake of these miRNAs at sites of injury might promote the proliferation of multiple cell types and stimulate the formation of new blood vessels for tissue repair.

While most of the focus on functional small RNAs in exosomes has been on the class of miRNAs, in contrast with what was previously reported for immune and neuronal cells $[28,63]$, MSC exosomes are highly enriched in tRNAs, specifically tRNA halves, and repeats compared with the producing cells.

Recent findings suggest that tRNA pools in proliferating cells versus differentiating cells are distinct. Importantly, it was noted that genes involved in cell-autonomous functions carry codons corresponding to proliferationassociated tRNAs, while genes linked to multicellularity require differentiation-associated tRNAs [75]. Therefore it would be very interesting to investigate the correlation between the most abundant tRNA species produced and released via exosomes by MSCs and the cellular protein composition, and how this might change upon activation of specific differentiation programs.

Post-transcriptional processing of tRNAs into tRNA fragments is a nonrandom evolutionary conserved mechanism [53, 54, 76]. Strikingly, tRNA fragments of defined sizes are highly represented in adult MSCs and tRNA halves appear to be massively sorted into MSC exosomes. The 14-30-nucleotide tRNA-derived RNA fragments (tRFs) are known to associate with Argonaute proteins and have similar properties to miRNAs [53]. Moreover, these fragments can define transformed cells [77]. The function of tRNA halves in mammalian cells, however, is largely unknown. Protein biosynthesis inhibition in response to stress conditions has been initially proposed as the most plausible explanation for tRNA cleavage. However, the two halves of the tRNA are usually unequally stable, suggesting more complex roles for these tRNA pieces [54]. More recently, 5' halves have been implicated in stress-induced translation inhibition and stress granule formation [78]. In MSCs it seems that tRNA halves are expressed at relatively high levels under standard culture conditions, possibly indicating physiological functions.

We found that ASC exosomes predominantly carry tRNA halves and are virtually devoid of full-length transcripts. BMSC exosomes have two different tRNA length profiles that seem to be related to differentiation status. Indeed, BMSCs expressing a high level of key stemness markers (BMSC II) package both full-length transcripts and 33-nucleotide fragments, while more differentiated cells (BMSC I) display the same tRNA length profile as ASC exosomes. Interestingly, however, the most abundant tRNAs in exosomes do not always correspond to those in cells, suggesting that cells can sort specific tRNAs perhaps as a mechanism for gene expression regulation. Analyzing the genomic coverage of the most abundant tRNA reads we found that ASC and BMSC I exosomes consistently display the $5^{\prime}$ halves of the most represented tRNA sequences, tRNA GCC (Gly) and CTC (Glu), respectively. The $5^{\prime}$ half of tRNA CTC (Glu) was recently shown to act in a miRNA/siRNA-like fashion to silence target mRNAs [56], although putative target transcripts for this and other fragments have not yet been identified.

The complementarity analysis of the most abundant tRNA species in cells and exosomes to the 3' UTRs of protein-coding genes highlighted interesting putative tRNA targets involved in stem cell renewal, differentiation, and immune modulation. For instance, we found: TFCP2L1, a transcription factor involved in stem cell self-renewal [79]; RUNX2 and SOX11, master transcription factors in MSC differentiation [80, 81]; SMAD3, mediator of TGF- $\beta$ induced proliferation, differentiation, and survival; RPS19, involved in erythropoietic differentiation and proliferation [82]; and immune-related factors and inflammation mediators such as HHLA2, EMR2, and TRIM62 [83-85]. Further studies will be required to elucidate the biological function of the tRNA fragments and to evaluate whether their release via exosomes is involved in orchestrating tissue architectures.

\section{Conclusions}

The role of EV-transferred RNA in physiological processes in vivo remains unclear, partly because the 
minimum amount of individual RNA molecules that is required for causing physiological changes in target cells is difficult to predict, and is likely to involve many factors. One recent hypothesis is that the most abundant and enriched RNA species in EVs play a dominant role. Using RNAseq analysis we could demonstrate that the most abundant and enriched small RNAs in adult MSC exosomes are defined tRNA species. Moreover, adipose and bone marrow MSC subtypes secrete different tRNA species that may be relevant for clinical applications. Future studies should focus on how these tRNA molecules are transported by MSC exosomes under physiological conditions and whether they influence their microenvironment in a cell type-dependent manner.

\section{Additional files}

Additional file 1: Figure S1. Shows the Bioinformatics workflow. Figure S2 shows MSC origin (A), expression of surface markers analyzed by FACS (B), and osteogenic differentiation assessed by Alizarin red staining (C). Figure S3 shows detection of CD63 and CD81 in MSC and exosomes, PL and FBS (A), and CDNA libraries of MSC cellular and exosomal RNA (B). Figure S4 shows correlation matrix of MSC and exosome samples based on the miRNA profiles (A), relative proportion of individual miRNAs in the repertoire of total miRNA reads in cells (B), and rpm of miRNAs differentially represented in cells and exosomes (C). Figure S5 shows relative distribution of tRNAs in MSCs, LCLs, and respective exosomes (A), differentially represented tRNAs in BMSC exosomes compared with ASC exosomes (B), and length distribution of the most represented tRNAs in MSC cells and exosomes (C). Figure S6 shows tRNAs differentially represented in exosomes compared with cells.

Additional file 2: Table S1. Presents antisense complementarity of the most abundant tRNA species to the 3' UTRs of annotated protein-coding transcripts.

\section{Abbreviations}

ALP: Alkaline phosphatase; ASC: Adipose-derived mesenchymal stem cell; BMSC: Bone marrow-derived mesenchymal stem cell; BSA: Bovine serum albumin; COL1A1: Collagen type 1 alpha 1; EEA1: Early endosome antigen A1; EM: Electron microscopy; EV: Extracellular vesicle; FACS: Fluorescenceactivated cell sorting; FBS: Fetal bovine serum; FITC: Fluorescein isothiocyanate; GAPDH: Glyceraldehyde 3-phosphate dehydrogenase; LCL: Lymphoblastoid cell; a-MEM: Alpha-minimum essential medium; MSC: Mesenchymal stem cell; miRNA: microRNA; MVB: Multivesicular body; PBS: Phosphate-buffered saline; PL: Platelet lysate; rpm: Reads per million; TGF- $\beta$ : Transforming growth factor beta; tRF: tRNA-derived RNA fragment; 3' UTR: 3' Untranslated region.

\section{Competing interests}

The authors declare that they have no competing interests.

\section{Authors' contributions}

SRB conceived and designed the study, performed the experiments, assembled and interpreted the data, and wrote the manuscript. KR analyzed and interpreted the data, performed the statistical analysis, and wrote the manuscript. DK-L, FJV, NZ, and MPL performed experiments. BN, FP, and HWMN isolated, characterized, and provided primary MSC cultures. NB conceived and designed the study. DMP conceived and designed the study, interpreted the data, and wrote the manuscript. All authors read and approved the final manuscript.

\section{Acknowledgments}

This work has been supported by the Italian Foundation for Cancer Research (FIRC) fellowship for Abroad, and the L'Oréal-UNESCO "For Women in Science" award (to SRB).

\section{Author details}

'Laboratory for Orthopedic Pathophysiology and Regenerative Medicine, Istituto Ortopedico Rizzoli, Bologna 40136, Italy. ${ }^{2}$ Department of Pathology, Cancer Center Amsterdam, VU University Medical Center, De Boelelaan 1117, 1081 HV Amsterdam, The Netherlands. ${ }^{3}$ Department of Biological Stress Response, Netherlands Cancer Institute, 1066 CX Amsterdam, The Netherlands. ${ }^{4}$ CNR — National Research Council of Italy, IGM, Bologna 40136, Italy. ${ }^{5} \mathrm{SC}$ Laboratory of Musculoskeletal Cell Biology, Istituto Ortopedico Rizzoli, Bologna 40136, Italy.

Received: 26 January 2015 Revised: 28 January 2015

Accepted: 15 June 2015 Published online: 01 July 2015

\section{References}

1. Farini A, Sitzia C, Erratico S, Meregalli M, Torrente Y. Clinical applications of mesenchymal stem cells in chronic diseases. Stem Cells Int. 2014;2014:306573.

2. Méndez-Ferrer S, Michurina TV, Ferraro F, Mazloom AR, Macarthur BD, Lira $\mathrm{SA}$, et al. Mesenchymal and haematopoietic stem cells form a unique bone marrow niche. Nature. 2010:466:829-34.

3. Dominici M, Le Blanc K, Mueller I, Slaper-Cortenbach I, Marini F, Krause D, et al. Minimal criteria for defining multipotent mesenchymal stromal cells. The International Society for Cellular Therapy position statement. Cytotherapy. 2006;8:315-7.

4. Pittenger MF, Mackay AM, Beck SC, Jaiswal RK, Douglas R, Mosca JD, et al. Multilineage potential of adult human mesenchymal stem cells. Science. 1999;284:143-7.

5. Johnson A, Dorshkind K. Stromal cells in myeloid and lymphoid long-term bone marrow cultures can support multiple hemopoietic lineages and modulate their production of hemopoietic growth factors. Blood. 1986;68:1348-54.

6. Schreml S, Babilas P, Fruth S, Orsó E, Schmitz G, Mueller MB, et al. Harvesting human adipose tissue-derived adult stem cells: resection versus liposuction. Cytotherapy. 2009;11:947-57.

7. Al-Nbaheen M, vishnubalaji R, Ali D, Bouslimi A, Al-Jassir F, Megges M, et al. Human stromal (mesenchymal) stem cells from bone marrow, adipose tissue and skin exhibit differences in molecular phenotype and differentiation potential. Stem Cell Rev Reports. 2013;9:32-43.

8. Bianco P, Cao X, Frenette PS, Mao JJ, Robey PG, Simmons PJ, et al. The meaning, the sense and the significance: translating the science of mesenchymal stem cells into medicine. Nat Med. 2013;19:35-42.

9. Ranganath $\mathrm{SH}$, Levy O, Inamdar MS, Karp JM. Harnessing the mesenchymal stem cell secretome for the treatment of cardiovascular disease. Cell Stem Cell. 2012;10:244-58.

10. Tögel F, Hu Z, Weiss K, Isaac J, Lange C, Westenfelder C. Administered mesenchymal stem cells protect against ischemic acute renal failure through differentiation-independent mechanisms. Am J Physiol Renal Physiol. 2005;289:F31-42.

11. Kordelas L, Rebmann V, Ludwig A-K, Radtke S, Ruesing J, Doeppner TR, et al. MSC-derived exosomes: a novel tool to treat therapy-refractory graft-versushost disease. Leukemia. 2014;28:970-3.

12. Baglio SR, Pegtel DM, Baldini N. Mesenchymal stem cell secreted vesicles provide novel opportunities in (stem) cell-free therapy. Front Physiol. 2012;3:359.

13. Binato R, de Souza FT, Lazzarotto-Silva C, Du Rocher B, Mencalha A, Pizzatt $\mathrm{L}$, et al. Stability of human mesenchymal stem cells during in vitro culture: considerations for cell therapy. Cell Prolif. 2013;46:10-22.

14. Wang Y, Zhang Z, Chi Y, Zhang Q, Xu F, Yang Z, et al. Long-term cultured mesenchymal stem cells frequently develop genomic mutations but do not undergo malignant transformation. Cell Death Dis. 2013;4:e950.

15. Behnan J, Isakson P, Joel M, Cilio C, Langmoen IA, Vik-Mo EO, et al. Recruited brain tumor-derived mesenchymal stem cells contribute to brain tumor progression. Stem Cells. 2014;32:1110-23.

16. Houthuijzen JM, Daenen LGM, Roodhart JML, Voest EE. The role of mesenchymal stem cells in anti-cancer drug resistance and tumour progression. Br J Cancer. 2012;106:1901-6.

17. Karnoub AE, Dash AB, Vo AP, Sullivan A, Brooks MW, Bell GW, et al. Mesenchymal stem cells within tumour stroma promote breast cancer metastasis. Nature. 2007:449:557-63.

18. Han I, Yun M, Kim E-O, Kim B, Jung M-H, Kim S-H. Umbilical cord tissue-derived mesenchymal stem cells induce apoptosis in $\mathrm{PC}-3$ prostate 
cancer cells through activation of JNK and downregulation of PI3K/AKT signaling. Stem Cell Res Ther. 2014;5:54.

19. Xu C, Lin L, Cao G, Chen Q, Shou P, Huang Y, et al. Interferon-a-secreting mesenchymal stem cells exert potent antitumor effect in vivo. Oncogene. 2014;33:5047-52.

20. Mittelbrunn M, Sánchez-Madrid F. Intercellular communication: diverse structures for exchange of genetic information. Nat Rev Mol Cell Biol. 2012;13:328-35

21. Lai RC, Arslan F, Lee MM, Sze NSK, Choo A, Chen TS, et al. Exosome secreted by MSC reduces myocardial ischemia/reperfusion injury. Stem Cell Res. 2010;4:214-22.

22. Valadi $H$, Ekström K, Bossios A, Sjöstrand M, Lee JJ, Lötvall JO. Exosome-mediated transfer of mRNAs and microRNAs is a novel mechanism of genetic exchange between cells. Nat Cell Biol. 2007;9:654-9.

23. Pegtel DM, Cosmopoulos K, Thorley-Lawson DA, van Eijndhoven MAJ, Hopmans ES, Lindenberg $J$, et al. Functional delivery of viral miRNAs via exosomes. Proc Natl Acad Sci U S A. 2010;107:6328-33.

24. Mittelbrunn M, Gutiérrez-Vázquez C, Villarroya-Beltri C, González S, Sánchez-Cabo F, González MÁ, et al. Unidirectional transfer of microRNA-loaded exosomes from T cells to antigen-presenting cells. Nat Commun. 2011;2:282.

25. Zhou W, Fong MY, Min Y, Somlo G, Liu L, Palomares MR, et al. Cancer-Secreted miR-105 destroys vascular endothelial barriers to promote metastasis. Cancer Cell. 2014;25:501-15.

26. Montecalvo A, Larregina AT, Shufesky WJ, Stolz DB, Sullivan MLG, Karlsson $J \mathrm{M}$, et al. Mechanism of transfer of functional microRNAs between mouse dendritic cells via exosomes. Blood. 2012;119:756-66.

27. Zhuang G, Wu X, Jiang Z, Kasman I, Yao J, Guan Y, et al. Tumour-secreted miR-9 promotes endothelial cell migration and angiogenesis by activating the JAK-STAT pathway. EMBO J. 2012;31:3513-23.

28. Nolte-'t Hoen ENM, Buermans HPJ, Waasdorp M, Stoorvogel W, Wauben MHM, 't Hoen PAC. Deep sequencing of RNA from immune cell-derived vesicles uncovers the selective incorporation of small non-coding RNA biotypes with potential regulatory functions. Nucleic Acids Res. 2012:40:9272-85

29. Hill AF, Pegtel DM, Lambertz U, Leonardi T, O'Driscoll L, Pluchino S, et al. ISEV position paper: extracellular vesicle RNA analysis and bioinformatics. J Extracell Vesicles. 2013;2. doi:10.3402/jev.v2i0.22859.

30. Xin H, Li Y, Buller B, Katakowski M, Zhang Y, Wang X, et al. Exosome-mediated transfer of miR-133b from multipotent mesenchymal stromal cells to neural cells contributes to neurite outgrowth. Stem Cells. 2012;30:1556-64.

31. Zhang H-C, Liu X-B, Huang S, Bi X-Y, Wang H-X, Xie L-X, et al. Microvesicles derived from human umbilical cord mesenchymal stem cells stimulated by hypoxia promote angiogenesis both in vitro and in vivo. Stem Cells Dev. 2012:21:3289-97.

32. Reis LA, Borges FT, Simões MJ, Borges AA, Sinigaglia-Coimbra R, Schor N. Bone marrow-derived mesenchymal stem cells repaired but did not prevent gentamicin-induced acute kidney injury through paracrine effects in rats. PLoS One. 2012;7:e44092.

33. Zhou Y, Xu H, Xu W, Wang B, Wu H, Tao Y, et al. Exosomes released by human umbilical cord mesenchymal stem cells protect against cisplatin-induced renal oxidative stress and apoptosis in vivo and in vitro. Stem Cell Res Ther. 2013;4:34.

34. Kim H-S, Choi D-Y, Yun SJ, Choi S-M, Kang JW, Jung JW, et al. Proteomic analysis of microvesicles derived from human mesenchymal stem cells. J Proteome Res. 2012;11:839-49.

35. Collino F, Deregibus MC, Bruno S, Sterpone L, Aghemo G, Viltono L, et al. Microvesicles derived from adult human bone marrow and tissue specific mesenchymal stem cells shuttle selected pattern of miRNAs. PLoS One. 2010;5:e11803.

36. Naaijkens BA, Niessen HWM, Prins H-J, Krijnen PAJ, Kokhuis TJA, de Jong N, et al. Human platelet lysate as a fetal bovine serum substitute improves human adipose-derived stromal cell culture for future cardiac repair applications. Cell Tissue Res. 2012;348:119-30.

37. De Korte D, Curvers J, de Kort WLAM, Hoekstra T, van der Poel CL, Beckers EAM, et al. Effects of skin disinfection method, deviation bag, and bacterial screening on clinical safety of platelet transfusions in the Netherlands. Transfusion. 2006;46:476-85.

38. Baglio SR, Devescovi V, Granchi D, Baldini N. MicroRNA expression profiling of human bone marrow mesenchymal stem cells during osteogenic differentiation reveals Osterix regulation by miR-31. Gene. 2013;527:321-31.
39. Verweij FJ, Van Eijndhoven MAJ, Middeldorp J, Pegtel DM. Analysis of viral microRNA exchange via exosomes in vitro and in vivo. Methods Mol Biol. 2013;1024:53-68.

40. Livak KJ, Schmittgen TD. Analysis of relative gene expression data using real-time quantitative PCR and the 2(-Delta Delta C(T)) method. Methods. 2001;25:402-8

41. Martin M. Cutadapt removes adapter sequences from high-throughput sequencing reads. EMBnet J. 2011;17:10-2.

42. Langmead B, Salzberg SL. Fast gapped-read alignment with Bowtie 2. Nat Methods. 2012:9:357-9.

43. Harrow J, Frankish A, Gonzalez JM, Tapanari E, Diekhans M, Kokocinski F, et al. GENCODE: the reference human genome annotation for The ENCODE Project. Genome Res. 2012;22:1760-74.

44. Griffiths-Jones S. The microRNA Registry. Nucleic Acids Res. 2004;32:D109-11.

45. Lowe TM, Eddy SR. tRNAscan-SE: a program for improved detection of transfer RNA genes in genomic sequence. Nucleic Acids Res. 1997;25:955-64.

46. Rosenkranz D, Zischler H. proTRAC - a software for probabilistic piRNA cluster detection, visualization and analysis. BMC Bioinformatics. 2012;13:5.

47. Karolchik D, Hinrichs AS, Furey TS, Roskin KM, Sugnet CW, Haussler D, et al. The UCSC Table Browser data retrieval tool. Nucleic Acids Res. 2004;32:D493-6.

48. Robinson MD, McCarthy DJ, Smyth GK. edgeR: a Bioconductor package for differential expression analysis of digital gene expression data. Bioinformatics. 2010;26:139-40.

49. Théry C, Amigorena S, Raposo G, Clayton A. Isolation and characterization of exosomes from cell culture supernatants and biological fluids. Curr Protoc Cell Biol. 2006; Chapter 3:Unit 3.22

50. Verweij FJ, van Eijndhoven MAJ, Hopmans ES, Vendrig T, Wurdinger T, Cahir-McFarland E, et al. LMP1 association with CD63 in endosomes and secretion via exosomes limits constitutive NF-kB activation. EMBO J. 2011;30:2115-29.

51. Koppers-Lalic D, Hackenberg M, Bijnsdorp I, van Eijndhoven M, Sadek P, Ylstra B, et al. Non-templated nucleotide additions distinguish the small RNA composition in cells from exosomes. Cell Rep. 2014;8:1649-58.

52. Li Z, Liu C, Xie Z, Song P, Zhao RCH, Guo L, et al. Epigenetic dysregulation in mesenchymal stem cell aging and spontaneous differentiation. PLoS One. 2011;6:e20526.

53. Kumar P, Anaya J, Mudunuri SB, Dutta A. Meta-analysis of tRNA derived RNA fragments reveals that they are evolutionarily conserved and associate with AGO proteins to recognize specific RNA targets. BMC Biol. 2014;12:78.

54. Gebetsberger J, Polacek N. Slicing tRNAs to boost functional ncRNA diversity. RNA Biol. 2013;10:1798-806.

55. Squadrito ML, Baer C, Burdet F, Maderna C, Gilfillan GD, Lyle R, et al. Endogenous RNAs modulate MicroRNA sorting to exosomes and transfer to acceptor cells. Cell Rep. 2014;8:1432-46.

56. Wang Q, Lee I, Ren J, Ajay SS, Lee YS, Bao X. Identification and functional characterization of tRNA-derived RNA fragments (tRFs) in respiratory syncytial virus infection. Mol Ther. 2013;21:368-79.

57. Yeo RWY, Lai RC, Zhang B, Tan SS, Yin Y, Teh BJ, et al. Mesenchymal stem cell: an efficient mass producer of exosomes for drug delivery. Adv Drug Deliv Rev. 2013;65:336-41.

58. Chen TS, Arslan F, Yin Y, Tan SS, Lai RC, Choo ABH, et al. Enabling a robust scalable manufacturing process for therapeutic exosomes through oncogenic immortalization of human ESC-derived MSCs. J Transl Med. 2011;9:47.

59. Balaj L, Lessard R, Dai L, Cho Y-J, Pomeroy SL, Breakefield XO, et al. Tumour microvesicles contain retrotransposon elements and amplified oncogene sequences. Nat Commun. 2011;2:180.

60. Demory Beckler M, Higginbotham JN, Franklin JL, Ham A-J, Halvey PJ, Imasuen IE, et al. Proteomic analysis of exosomes from mutant KRAS colon cancer cells identifies intercellular transfer of mutant KRAS. Mol Cell Proteomics. 2013;12:343-55.

61. Kim DH, Yoo KH, Choi KS, Choi J, Choi S-Y, Yang S-E, et al. Gene expression profile of cytokine and growth factor during differentiation of bone marrow-derived mesenchymal stem cell. Cytokine. 2005;31:119-26.

62. Hoch Al, Binder BY, Genetos DC, Leach JK. Differentiation-dependent secretion of proangiogenic factors by mesenchymal stem cells. PLoS One. 2012:7:e35579.

63. Bellingham SA, Coleman BM, Hill AF. Small RNA deep sequencing reveals a distinct miRNA signature released in exosomes from prion-infected neuronal cells. Nucleic Acids Res. 2012;40:10937-49. 
64. Vojtech L, Woo S, Hughes S, Levy C, Ballweber L, Sauteraud RP, et al. Exosomes in human semen carry a distinctive repertoire of small noncoding RNAs with potential regulatory functions. Nucleic Acids Res. 2014;42:7290-304.

65. Chen TS, Lai RC, Lee MM, Choo ABH, Lee CN, Lim SK. Mesenchymal stem cell secretes microparticles enriched in pre-microRNAs. Nucleic Acids Res. 2010;38:215-24.

66. Clark EA, Kalomoiris S, Nolta JA, Fierro FA. Concise review: MicroRNA function in multipotent mesenchymal stromal cells. Stem Cells. 2014;32:1074-82.

67. Kim YJ, Hwang SH, Lee SY, Shin KK, Cho HH, Bae YC, et al. miR-486-5p induces replicative senescence of human adipose tissue-derived mesenchymal stem cells and its expression is controlled by high glucose. Stem Cells Dev. 2012;21:1749-60.

68. Zhao X, Liu D, Gong W, Zhao G, Liu L, Yang L, et al. The toll-like receptor 3 ligand, poly(l:C), improves immunosuppressive function and therapeutic effect of mesenchymal stem cells on sepsis via inhibiting MiR-143. Stem Cells. 2014;32:521-33.

69. Li J, Dong J, Zhang Z-H, Zhang D-C, You X-Y, Zhong Y, et al. miR-10a restores human mesenchymal stem cell differentiation by repressing KLF4. J Cell Physiol. 2013;228:2324-36.

70. Huang S, Wang S, Bian C, Yang Z, Zhou H, Zeng Y, et al. Upregulation of miR-22 promotes osteogenic differentiation and inhibits adipogenic differentiation of human adipose tissue-derived mesenchymal stem cells by repressing HDAC6 protein expression. Stem Cells Dev. 2012;21:2531-40.

71. Zhang F, Jing S, Ren T, Lin J. microRNA-10b promotes the migration of mouse bone marrow-derived mesenchymal stem cells and downregulates the expression of E-cadherin. Mol Med Rep. 2013;8:1084-8.

72. Nagpal N, Kulshreshtha R. miR-191: an emerging player in disease biology. Front Genet. 2014;5:99

73. Urbich C, Kuehbacher A, Dimmeler S. Role of microRNAs in vascular diseases, inflammation, and angiogenesis. Cardiovasc Res. 2008;79:581-8.

74. Yoo JK, Kim J, Choi S, Noh HM, Kwon YD, Yoo H, et al. Discovery and characterization of novel microRNAs during endothelial differentiation of human embryonic stem cells. Stem Cells Dev. 2012;21:2049-57.

75. Gingold H, Tehler D, Christoffersen NR, Nielsen MM, Asmar F, Kooistra SM, et al. A dual program for translation regulation in cellular proliferation and differentiation. Cell. 2014;158:1281-92.

76. Lee YS, Shibata Y, Malhotra A, Dutta A. A novel class of small RNAs: tRNA-derived RNA fragments (tRFs). Genes Dev. 2009;23:2639-49.

77. Maute RL, Schneider C, Sumazin P, Holmes A, Califano A, Basso K, et al. tRNA-derived microRNA modulates proliferation and the DNA damage response and is down-regulated in B cell lymphoma. Proc Natl Acad Sci U S A. 2013;110:1404-9.

78. Emara MM, Ivanov P, Hickman T, Dawra N, Tisdale S, Kedersha N, et al. Angiogenin-induced tRNA-derived stress-induced RNAs promote stress-induced stress granule assembly. J Biol Chem. 2010;285:10959-68.

79. Ye S, Li P, Tong C, Ying Q-L. Embryonic stem cell self-renewal pathways converge on the transcription factor Tfcp2l1. EMBO J. 2013;32:2548-60.

80. Komori T. Regulation of bone development and extracellular matrix protein genes by RUNX2. Cell Tissue Res. 2010;339:189-95.

81. Gadi J, Jung S-H, Lee M-J, Jami A, Ruthala K, Kim K-M, et al. The transcription factor protein Sox11 enhances early osteoblast differentiation by facilitating proliferation and the survival of mesenchymal and osteoblast progenitors. J Biol Chem. 2013;288:25400-13.

82. Bibikova E, Youn M-Y, Danilova N, Ono-Uruga $Y$, Konto-Ghiorghi $Y$, Ochoa R, et al. TNF-mediated inflammation represses GATA1 and activates p38 MAP kinase in RPS19-deficient hematopoietic progenitors. Blood. 2014; 124:3791-8.

83. Zhao R, Chinai JM, Buhl S, Scandiuzzi L, Ray A, Jeon H, et al. HHLA2 is a member of the B7 family and inhibits human CD4 and CD8 T-cell function. Proc Natl Acad Sci U S A. 2013;110:9879-84.
84. Yona S, Lin H-H, Dri P, Davies JQ, Hayhoe RPG, Lewis SM, et al. Ligation of the adhesion-GPCR EMR2 regulates human neutrophil function. FASEB J. 2008;22:741-51.

85. Uchil PD, Hinz A, Siegel S, Coenen-Stass A, Pertel T, Luban J, et al. TRIM protein-mediated regulation of inflammatory and innate immune signaling and its association with antiretroviral activity. J Virol. 2013;87:257-72.

\section{Submit your next manuscript to BioMed Central and take full advantage of:}

- Convenient online submission

- Thorough peer review

- No space constraints or color figure charges

- Immediate publication on acceptance

- Inclusion in PubMed, CAS, Scopus and Google Scholar

- Research which is freely available for redistribution 